ARTICLE

https://doi.org/10.1038/s41467-020-20718-8

\title{
The complex structure of GRL0617 and SARS-CoV-2 PLpro reveals a hot spot for antiviral drug discovery
}

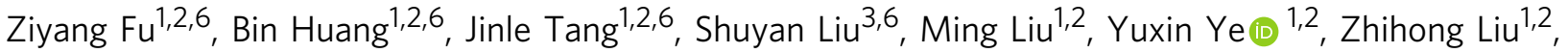 \\ Yuxian Xiong ${ }^{1,2}$, Wenning Zhu',2, Dan Cao ${ }^{1,2}$, Jihui $\mathrm{Li}^{1,2}$, Xiaogang Niu ${ }^{4}$, Huan Zhou ${ }^{5}$, Yong Juan Zhao', \\ Guoliang Zhang (i) ${ }^{3 凶}$ \& Hao Huang (1) ${ }^{1,2}{ }^{凶}$
}

SARS-CoV-2 is the pathogen responsible for the COVID-19 pandemic. The SARS-CoV-2 papain-like cysteine protease (PLpro) has been implicated in playing important roles in virus maturation, dysregulation of host inflammation, and antiviral immune responses. The multiple functions of PLpro render it a promising drug target. Therefore, we screened a library of approved drugs and also examined available inhibitors against PLpro. Inhibitor GRL0617 showed a promising in vitro $\mathrm{IC}_{50}$ of $2.1 \mu \mathrm{M}$ and an effective antiviral inhibition in cell-based assays. The co-crystal structure of SARS-CoV-2 PLproC1115 in complex with GRL0617 indicates that GRL0617 is a non-covalent inhibitor and it resides in the ubiquitinspecific proteases (USP) domain of PLpro. NMR data indicate that GRL0617 blocks the binding of ISG15 C-terminus to PLpro. Using truncated ISG15 mutants, we show that the Cterminus of ISG15 plays a dominant role in binding PLpro. Structural analysis reveals that the ISG15 C-terminus binding pocket in PLpro contributes a disproportionately large portion of binding energy, thus this pocket is a hot spot for antiviral drug discovery targeting PLpro.

\footnotetext{
${ }^{1}$ State Key Laboratory of Chemical Oncogenomics, School of Chemical Biology and Biotechnology, Peking University Shenzhen Graduate School, Shenzhen 518055, China. ${ }^{2}$ Laboratory of Structural Biology and Drug Discovery, Peking University Shenzhen Graduate School, Shenzhen 518055, China. ${ }^{3}$ National Clinical Research Center for Infectious Diseases, Shenzhen Third People's Hospital, Southern University of Science and Technology, Shenzhen 518112, China. ${ }^{4}$ College of Chemistry and Molecular Engineering, Beijing Nuclear Magnetic Resonance Center, Peking University, Beijing 100871, China. ${ }^{5}$ Shanghai Advanced Research Institute, Chinese Academy of Sciences, Shanghai, China. ${ }^{6}$ These authors contributed equally: Ziyang Fu, Bin Huang, Jinle Tang,

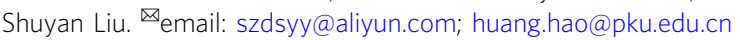


T he COVID-19 pandemic has caused devastating damage to the world and it has resulted in over 12 million confirmed cases and over half a million deaths as of July $14,2020^{1}$. The novel SARS-CoV-2 coronavirus is the etiological agent responsible for the pandemic, and it belongs to the beta coronavirus family ${ }^{2-4}$. Similar to the two beta coronaviruses, SARS and MERS, which have caused pandemic or epidemic in human history, the novel SARS-CoV-2 also causes severe acute respiratory syndromes ${ }^{5,6}$. Unexpectedly, SARS-CoV-2 has been reported to have more mild symptoms but much higher transmission rate $^{7,8}$, therefore it has caused the biggest catastrophe to the world healthcare since the Spanish flu in $1918-1920^{9}$. Four previously deemed promising antiviral drugs, i.e., Remdesivir, Hydroxychloroquine, Lopinavir, and Interferon, showed no or little effect on hospitalized COVID-19 patients, in WHO global solidarity clinical trials ${ }^{10}$. Encouragingly, the UK started rollout of an mRNA vaccine developed by Pfizer/BioNtech in early December 2020, with its long-term safety and efficacy to be assessed. Meanwhile, progress has been made in the discovery of antibodies for COVID-19. Therefore, anti-SARS-CoV-2 drugs are urgently needed.

As a positive strand RNA virus, SARS-CoV-2 encodes two functional proteases, i.e., the papain-like protease (PLpro) and the 3-chymotrypsin-like cysteine protease (Mpro or 3CLpro). The major function of Mpro is to cleave the viral polyproteins, which is critical for virus maturation, replication, and invasion. Several potent Mpro covalent inhibitors have been reported and their cocrystal structures provided potential opportunities for structurebased drug optimization ${ }^{11-14}$. SARS-CoV PLpro is a cysteine protease with multiple major functions, including processing of the viral polyprotein chain for viral protein maturation, dysregulating host inflammation responses through deubiquitylation, and impairing the host type I interferon antiviral immune responses by removing interferon stimulated gene 15 (ISG15) modifications ${ }^{15-17}$. ISG15 modification (ISGylation) is the covalent conjugation of ISG15 protein (MW $=17.1 \mathrm{kDa})$ to protein substrates and this process is known to inhibit virus replication ${ }^{18-20}$. SARS-CoV-2 PLpro $(\mathrm{MW}=35.6 \mathrm{kDa})$, sharing $\sim 83 \%$ sequence identity with SARS-CoV PLpro, contains an Nterminal ubiquitin-like (UBL) domain and a C-terminal ubiquitin-specific protease (USP) domain with implicated catalytic functions of cleaving ubiquitin (Ub) or ISG15 modifications from host proteins ${ }^{21}$.

Besides Mpro, PLpro has been considered another potentially promising target for drug discovery to treat COVID-1922. Due to the urgent need of therapies for the pandemic, the strategy of repurposing approved drugs or optimizing new compounds has been employed to fight COVID-1914,23-25. Accordingly, we describe our efforts in screening of a compound library of drugs approved by FDA or CFDA (China FDA) against SARS-CoV-2 PLpro, and structural characterization of the interactions between a promising drug lead and PLpro. The co-crystal structure of PLpro in complex with the compound GRL0617 and its antiviral effect provided direct proof of druggability of PLpro and the mechanism of action of the compound. Further structural and biophysical analysis reveals that the C-terminus of ISG15 plays a dominant role in its binding with PLpro through extensive hydrogen bonds and electrostatic interactions. Therefore, the ISG15-C-terminus binding cleft in PLpro is a hot spot for antiviral drug discovery.

\section{Results}

High-throughput screening of a library of approved drugs against PLpro. To repurpose existing drugs to inhibit the SARSCoV-2 PLpro, we initiated screening of a 2040-compound library of drugs approved by FDA or CFDA against SARS-CoV-2 PLpro (Supplementary Table 1). First, we set up a FRET assay to characterize the enzymatic activity of SARS-CoV-2 PLpro based on an established assay for SARS-CoV PLpro ${ }^{26}$. The recombinant full-length SARS-CoV-2 PLpro protein was expressed in Escherichia coli, and subsequently purified using his-tag chromatography and size exclusion chromatography (Supplementary Fig. 1). A commercially available fluorogenic peptide substrate Arg-Leu-Arg-Gly-Gly-AMC (RLRGG-AMC), representing the Cterminal residues of ubiquitin, was used to report the enzymatic activity of PLpro. The first round of screening provided $\sim 30$ compounds with over $50 \%$ inhibition at $100 \mu \mathrm{M}$. Because the FDA approved drug Tioguanine (6-TG) has been previously tested on SARS-CoV and MERS PLpro proteins with effective inhibitions $^{27,28}$, we determined its potency against SARS-CoV-2 PLpro and the $\mathrm{IC}_{50}$ value was $72 \pm 12 \mu \mathrm{M}$, so we used it as a positive control throughout the screening. Hits from the first round of screening went into the second round of validation using the same enzymatic assay. After removing compounds with poor solubility, strong reactivity, or high intrinsic fluorescence, seven relatively potent compounds including 6-TG were measured for $\mathrm{IC}_{50}$. These seven drugs showed modest $\mathrm{IC}_{50}$ values ranging from 29 to $91 \mu \mathrm{M}$ (Supplementary Fig. 3). Although these compounds can potentially provide a starting point for further optimization, their low potency implies a need of large amounts of resources and time input.

Identification of GRL0617 as an inhibitor for SARS-CoV-2 PLpro. Parallelly, we cherry-picked GRL0617 and its analog compound 6 from promising SARS-CoV PLpro inhibitors ${ }^{26,29}$ based on high sequence identity between the SARS-CoV and SARS-CoV-2 PLpro proteins (Supplementary Fig. 2). The in vitro $\mathrm{IC}_{50}$ values of GRL0617 and compound 6 against SARS-CoV-2 PLpro were $2.1 \pm 0.2 \mu \mathrm{M}$ and $11 \pm 3 \mu \mathrm{M}$, respectively (Fig. 1a). The compound 6, as an acetamide derivative of GRL0617, did not show improved potency in the in vitro FRET assay. Our data suggested that GRL0617 is a promising lead compound and therefore it was subjected to further antiviral, structural, and mechanistic studies. Our identification of GRL0617 and its analogs along with their potencies are in line with recent studies by the Pegan group ${ }^{30}$, the Dikic group ${ }^{31}$, and the Komander group ${ }^{32}$.

Inhibition of the in-cell deubiquitinating and deISGylating activity of PLpro by GRL0617. To assess whether GRL0617 can inhibit the in cyto deubiquitinating and deISGylating activity of SARS-CoV-2 PLpro, we transfected HEK293T cells with plasmids of PLpro and the ISGylation machinery (Ube1L, UbcH8, HECR5, and ISG15) and then treated with GRL0617 at different concentrations for $24 \mathrm{~h}$. Our data (Fig. 1b) showed that the deubiquitinating activity of SARS-CoV-2 PLpro is weak but its deISGylating activity is relatively strong, which is consistent with recent publications ${ }^{30-33}$. SARS-CoV-2 PLpro is capable of reversing the ISGylation and polyubiquitination (to a much lesser extent) of cellular substrates (Fig. 1b). Furthermore, addition of GRL0617 caused inhibition of SARS-CoV-2 PLpro and resulted in partially recovered poly-ubiquitin-conjugates and ISG15conjugates (Fig. 1b). Moreover, we further used interferon $\beta$ (IFN- $\beta$ ) to induce the ISGylation in HEK293T cells which generated a remarkable amount of ISG15-conjugated cellular substrates (Fig. 1c, lanes 1 and 2). The addition of $160 \mu \mathrm{M}$ of GRL0617 alone to cell lysate had no observable effect to ISGylation (Fig. 1c, lanes 2 and 3), which demonstrated the selectivity of the compound over other deISGylating enzymes in cells, such as USP18. Indeed, GRL0617 showed no inhibition effect on the 

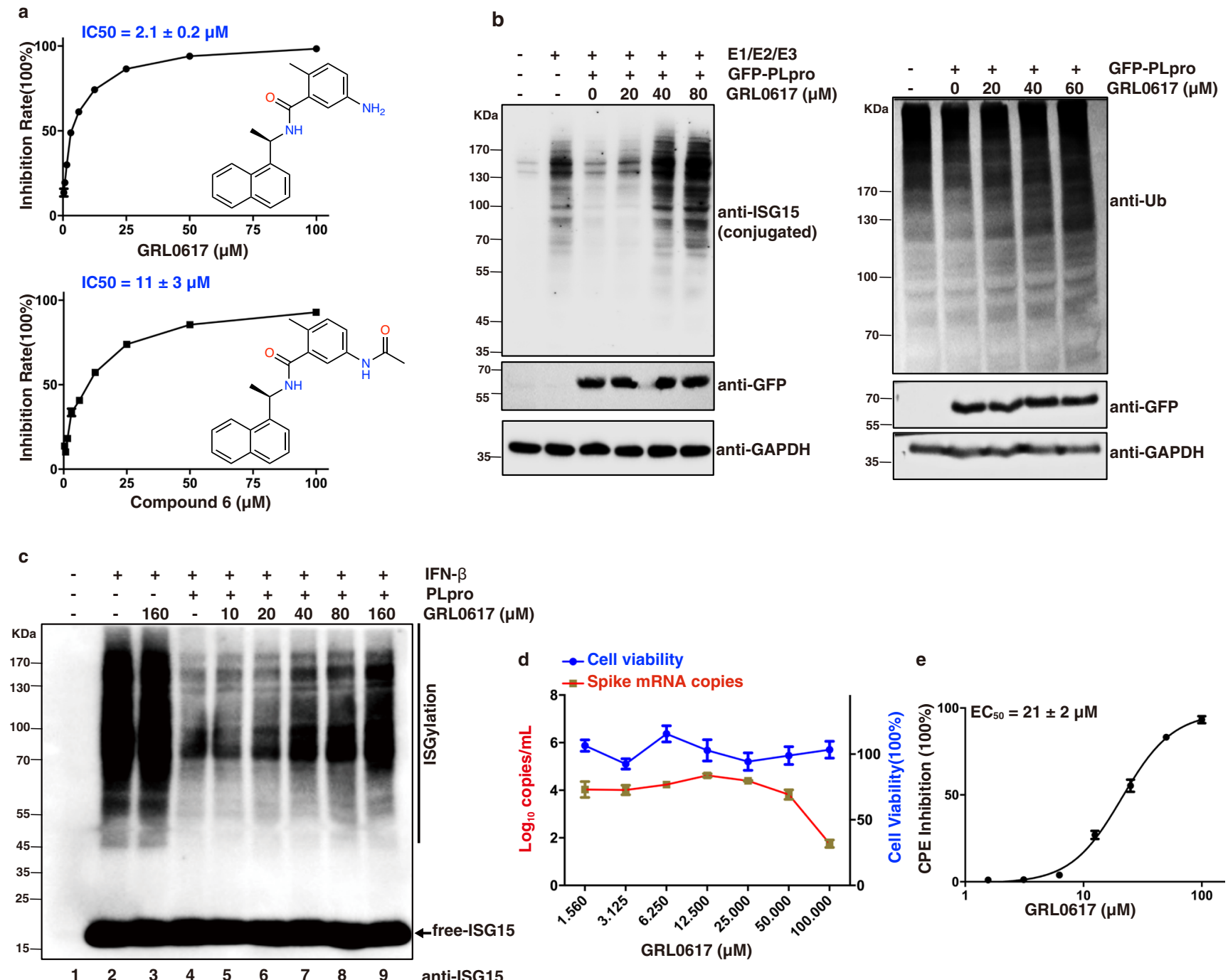

Fig. 1 Inhibitory activity of GRL0617 against SARS-CoV-2 PLpro. a The inhibitory activity of GRL0617 and compound 6 against PLpro was measured using the peptide RLRGG-AMC as a substrate. IC 50 was presented as mean \pm SEM, $n=3$ independent experiments. $\mathbf{b}$ In-cell delSGylating (left) and deubiquitinating (right) activities of PLpro, HEK293T cells were transfected for $24 \mathrm{~h}$ with plasmids encoding GFP-PLpro, ISG15, and E1(Ube1L)/E2(UbcH8)/ E3(HECR5) enzymes, alone or in combination. Cells were treated for an additional $24 \mathrm{~h}$ with indicated concentrations of GRL0617. Cell lysates were subjected to immunoblotting with anti-ubiquitin, anti-ISG15, and anti-GFP antibodies. GAPDH served as a loading control. A representative from three independent experiments is shown. $\mathbf{c}$ HEK293T cells were treated with or without $500 \mathrm{U} / \mathrm{mL}$ interferon $\beta$ (IFN- $\beta$ ) for $48 \mathrm{~h}$. The cell extracts were incubated with purified recombinant PLpro $(100 \mathrm{nM})$ and indicated concentrations of GRLO617 for $60 \mathrm{~min}$ at $37^{\circ} \mathrm{C}$, followed by immunoblotting analysis with antiISG15. A representative from three independent experiments is shown. d Antiviral activity of GRL0617 on SARS-CoV-2 and the cytotoxicity of GRL0617 on Vero E6 cells. Vero E6 cells were infected with SARS-CoV-2 using a multiplicity-of-infection (MOI) of 0.01. The quantification of absolute viral RNA copies (per $\mathrm{mL}$ ) in the supernatant at $48 \mathrm{~h}$ post-infection was determined by qRT-PCR analysis. The cytotoxicity of GRL0617 on Vero E6 cells was measured using CCK8. All data are shown as mean $\pm \mathrm{SEM}, n=3$ independent experiments. e EC 50 was presented as mean $\pm \mathrm{SEM}, n=3$ independent experiments, the cytopathic effect (CPE) analysis revealed an $\mathrm{EC}_{50}$ of $21 \pm 2 \mu \mathrm{M}$.

recombinant mouse USP18 protein (Supplementary Fig. 5g), which is consistent with other studies ${ }^{26,31}$. Further addition of purified SARS-CoV-2 PLpro (100 nM) to cell lysate efficiently reduced ISG15-conjugated proteins (Fig. 1c, lanes 2 and 4). In contrast, addition of GRL0617 to cell lysate together with PLpro recovered the ISGylation in a dose-dependent manner (Fig. 1c, lanes 5-9) with significant band recovery seen at $40 \mu \mathrm{M}$ of GRL0617. Clearly, GRL0617 inhibited the deISGylation activity of PLpro through an on-target effect.

Antiviral activity of the inhibitor GRL0617. To further validate the potential of PLpro as an antiviral drug target, we tested GRL0617 for its inhibitory activity in Vero E6 cells infected with SARS-CoV-2 at a multiplicity-of-infection (MOI) of 0.01. The
mRNA copy numbers of the viral spike protein were monitored to evaluate antiviral activity of the compound. Based on the dosedependent response, GRL0617 showed a clear inhibition of viral replication and $100 \mu \mathrm{M}$ of GRL0617 resulted in over 50\% inhibition. No apparent cytotoxicity on Vero E6 cells was observed in our assay with concentrations up to $100 \mu \mathrm{M}$ (Fig. 1d). The cytopathic effect (CPE) analysis revealed an $\mathrm{EC}_{50}$ of $21 \pm 2 \mu \mathrm{M}$ (Fig. 1e). This in cyto antiviral potency of GRL0617 is also in line with other recent studies ${ }^{30,31}$

The co-crystal structure of SARS-CoV-2 PLpro in complex with GRL0617. A co-crystal structure would be crucial to understand the mechanism of inhibition of SARS-CoV-2 by GRL0617, therefore we set out to solve the co-crystal structure. 
a 1
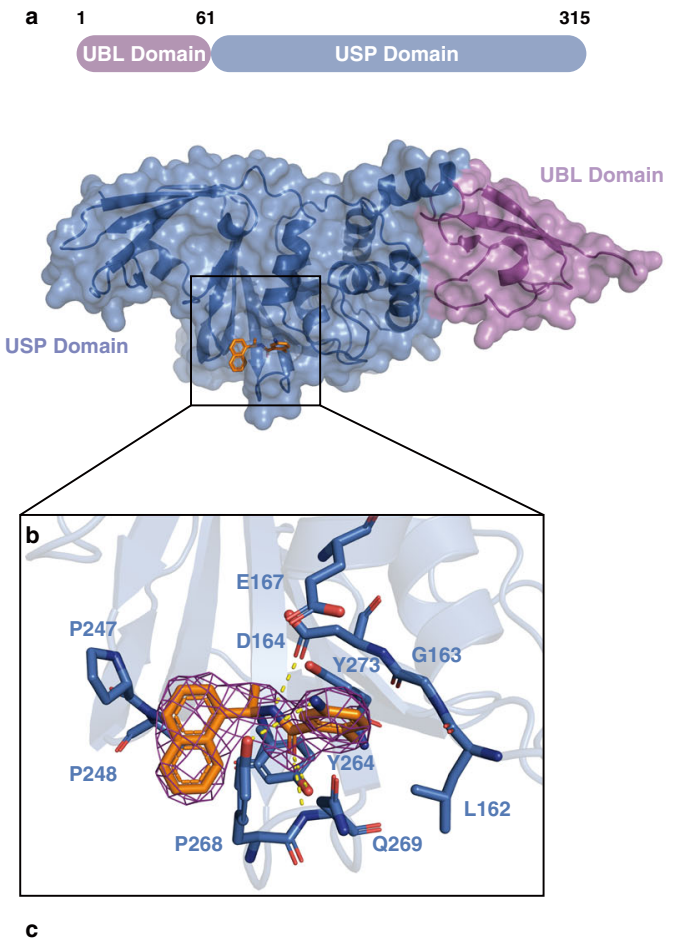

d

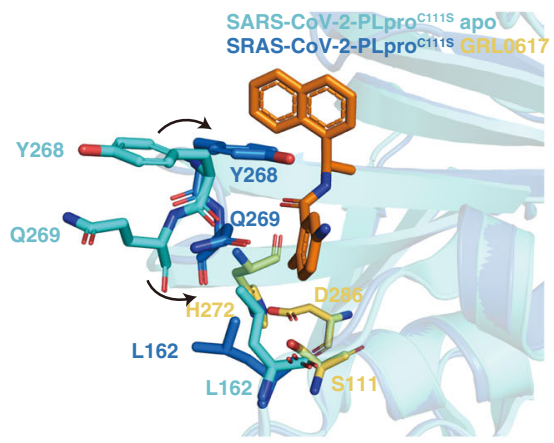

e
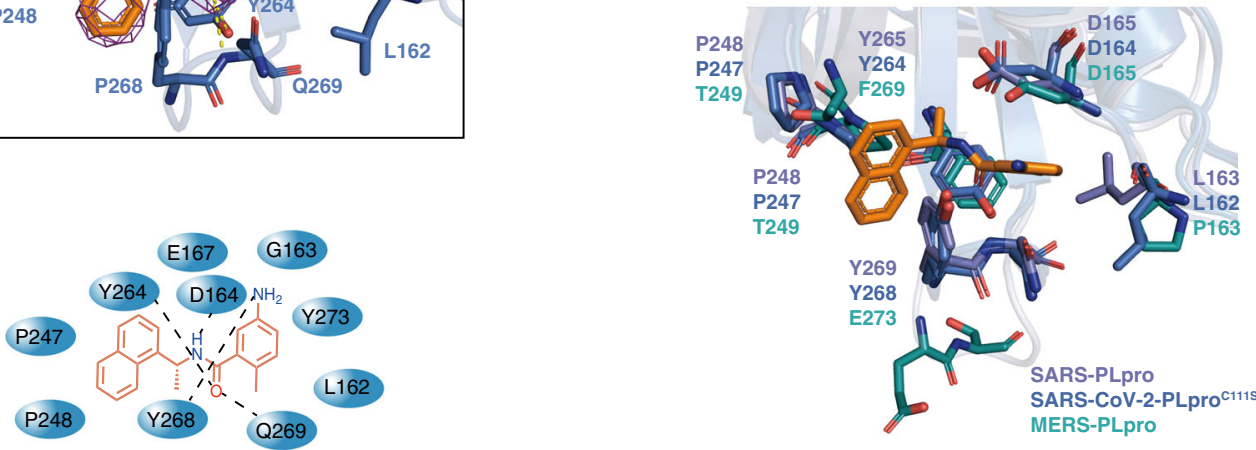

Fig. 2 Structural and mechanistic analysis of SARS-CoV-2 PLproC111S in complex with GRL0617, and in comparison with SARS-CoV and MERS PLpro. a Surface and cartoon structures of SARS-CoV-2 PLpro in complex with GRL0617 (orange sticks) showing the N-terminal UBL domain (magenta) and Cterminal USP domain (marine). b The binding pocket of GRL0617 in PLpro. The PLpro residues involved in GRL0617 binding are shown as marine sticks. The 2Fo-Fc omit map (contour level $=1.6 \sigma$, shown as purple mesh). c Schematic diagram of SARS-CoV-2 PLpro C111S/GRL0617 interactions shown in (b). d Comparison of GRL0617-bound (marine) PLproC111S and unbound (cyan) PLproC111S (PDB ID: 6WRH [https://doi.org/10.2210/pdb6wrh/pdb]) structures. Y268 and Q269 on the BL2 loop shifted toward GRL0617 upon binding. GRL0617 shown as orange sticks; the catalytic triad residues (S111 in place of $\mathrm{C111}, \mathrm{H} 272$, and D286) are shown in yellow; Y268 and Q269 are shown in marine in bound state, and in cyan in unbound state. e Comparisons of the binding sites of SARS-CoV PLpro/GRL0617 (slate sticks, PDB ID: 3E9S [https://doi.org/10.2210/pdb3e9s/pdb])26, SARS-CoV-2 PLproC111S/GRL0617 (marine sticks), and MERS-CoV PLpro (deep teal sticks, PDB ID: 4RNA [https://doi.org/10.2210/pdb4rna/pdb]) ${ }^{37}$.

However, the crystals of wild-type SARS-CoV-2 PLpro were difficult to grow. Therefore, we turned to grow co-crystals of SARS-CoV-2 PLpro ${ }^{\text {C111S }}$ in complex with GRL0617 by incubating the compound with the protein before setting up crystal trays. The obtained co-crystal diffracted at $3.2 \AA$ (Fig. $2 \mathrm{a}-\mathrm{e}$ and Supplementary Table 2). When our manuscript was in the review process, a few groups also reported the co-crystal structures of SARS-CoV-2 PLpro and GRL0617 or its analogs ${ }^{34,35}$. The crystal of PLpro/GRL0617 belongs to the space group $\mathrm{I}_{1} 22$ with one protein molecule in each asymmetric unit. SARS-CoV-2 PLpro has two domains, i.e., the N-terminal UBL domain and the Cterminal USP domain (Fig. 2a). Based on the B factor analysis, the palm and thumb regions in the USP domain have lower B factor values than the UBL domain and the fingers region of the USP domain, indicating that the palm and thumb regions are relatively more rigid than rest of the structure. As shown in the $2 \mathrm{Fo}-\mathrm{Fc}$ electron density map (Fig. 2a, b) as well as in the difference Fo-Fc electron density map (Supplementary Fig. 4b), GRL0617 resides in a pocket in the palm region of PLpro. GRL0617 is apart from the catalytical triad (including S111 in place of C111, H272, and D286) of PLpro with a minimum distance of $7.5 \AA$ to S111 (from the methyl of the 4-Methylbenzenamine moiety of GRL0617 to the sidechain oxygen of S111). Therefore, GRL0617 inhibits SARS-CoV-2 PLpro in a non-covalent manner. The GRL0617bound PLpro structure is overall similar to the available apostructure of PLpro ${ }^{\mathrm{C} 111}$ (PDB 6WRH) with a backbone RMSD of $0.76 \AA$, except for two residues on the BL2 loop, i.e., Y268 and Q269 (Fig. 2d). Upon binding to GRL0617, the sidechains of Y268 and Q269 shifted toward GRL0617 to form polar and hydrophobic interactions with the compound and stabilized its binding (Fig. 2d). Specifically, the sidechain oxygen of Y268 forms a hydrogen bond with the amino group on the benzene ring of GRL0617, and another hydrogen bond of the backbone amino group of Q269 with the carbonyl oxygen of GRL0617 (Fig. 2b, c). In comparison with the apo-wild-type (wt-, PDB 6W9C [https://doi.org/10.2210/pdb6w9c/pdb]) or C111S (PDB 6WRH [https://doi.org/10.2210/pdb6wrh/pdb]) structures of SARS-CoV-2 PLpro, the BL2 loop in GRL0617-bound PLpro structure shifted toward the compound to form a T-shaped $\pi-\pi$ stacking with the naphthalene group of GRL0617 and it also formed a deeper pocket to better accommodate the compound (Fig. 2e and Supplementary Fig. 5a, d, e). Other polar interactions 
include the hydrogen bonds between D164 and the amide NH of GRL0617, as well as between Y264 and the carbonyl oxygen of GRL0617. In addition, hydrophobic integration also contributed to the binding of GRL0617 to PLpro, e.g., the naphthalene group of GRL0617 is involved in the interactions with aromatic residues Y264 and Y268, and the hydrophobic sidechains of P247 and P248 (Fig. 2b, c).

Since GRL0617 is capable of inhibiting both SARS-CoV and SARS-CoV-2, it is of interest to understand its selectivity on coronaviral PLpro proteins from a structural biology perspective. It has been reported that naphthalene-based compounds have low to zero potency toward MERS PLpro ${ }^{36,37}$. The superposition of GRL0617 on a surface model of the MERS PLpro structure ${ }^{37}$ indicated that the original pocket in MERS PLpro might be too shallow to allow GRL0617 to bind with extensive contacts, and the naphthalene moiety of GRL0617 would also be in a steric clash with T249 of MERS PLpro (Supplementary Fig. 5c). In contrast to SARS and SARS-CoV-2, the BL-2 loop of MERS is one residue longer, but it lacks the critical Y268 of SARS-CoV-2 which played a critical role in encircling GRL0617 in the cocrystal structure (Supplementary Fig. 2). The extra residue of MERS PLpro may rearrange the hydrogen-bond interaction network of the BL2 loop and the lack of the aromatic tyrosine clearly resulted in the removal of the T-shaped $\pi-\pi$ stacking and van der Waals interactions with the naphthalene group of GRL0617 (Fig. 2e and Supplementary Fig. 5a-e). Our enzymatic assay confirmed the lack of inhibition of MERS PLpro by GRL0617 or compound 6, which is consistent with our structural analysis (Supplementary Fig. 5f) and a recent study ${ }^{31}$.

GRL0617 is a protein-protein interaction (PPI) inhibitor revealed by NMR. Because GRL0617 is a non-covalent inhibitor binding in the USP domain, i.e., the catalytic domain of SARSCoV-2 PLpro, it is of interest to see if GRL0617 would disrupt the interactions between ISG15 or Ub and PLpro. Solution state nuclear magnetic resonance (NMR) was employed to characterize the binding of ISG15 or Ub to PLpro as well as the perturbation of their bindings by GRL0617. 2-D NMR ${ }^{1} \mathrm{H},{ }^{15} \mathrm{~N}$-HSQC spectrum of ${ }^{15} \mathrm{~N}$-ISG15 (0.1 mM) showed typical features for a wellfolded protein with well-dispersed cross peaks (Fig. 3a). The addition of $0.15 \mathrm{mM}$ SARS-CoV-2 PLpro into $0.1 \mathrm{mM}{ }^{15} \mathrm{~N}$-ISG15 caused drastic peak broadening and peak intensity loss, which is a characteristic of PPIs in the intermediate chemical exchange regime (Fig. 3b). Increasing concentrations of GRL0617 were a

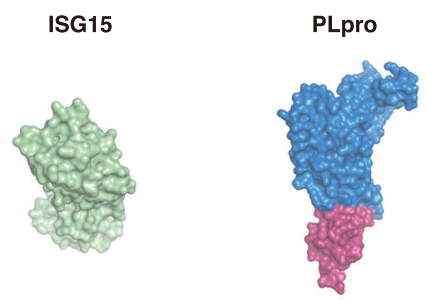

$0.1 \mathrm{mM}{ }^{15} \mathrm{~N}-$ ISG15 b

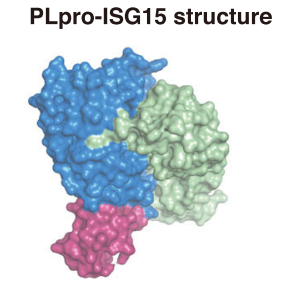

$0.1 \mathrm{mM}{ }^{15} \mathrm{~N}-$-ISG15

+ 0.15 mM PLpro
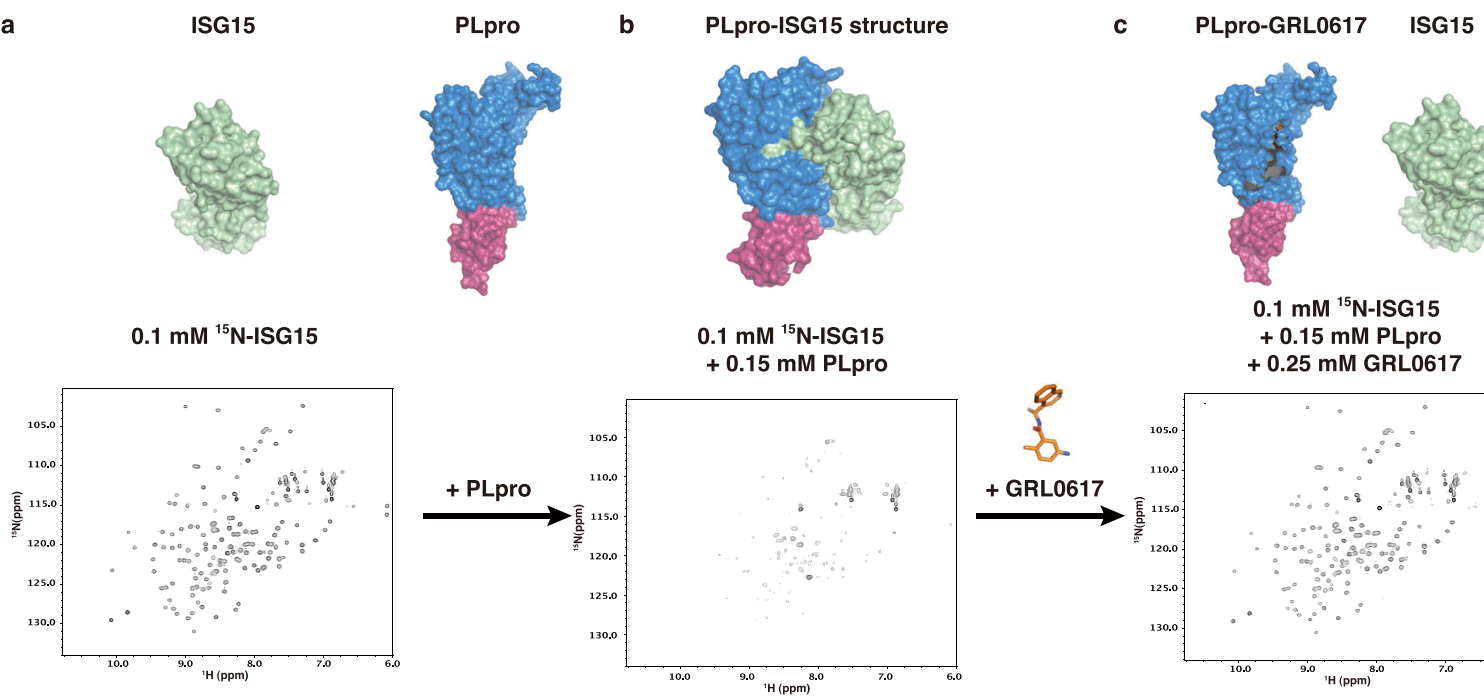

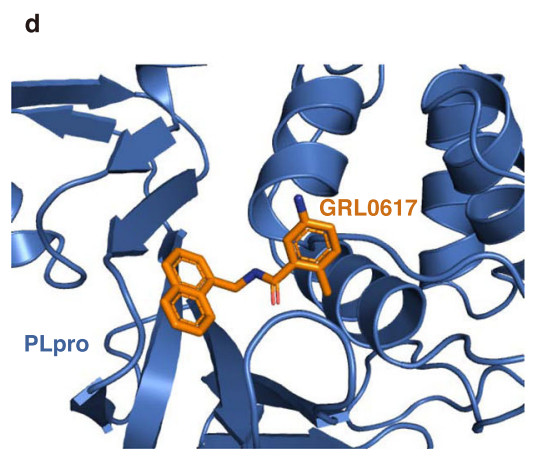

SARS-CoV-2-PLproc111s-GRL0617 e

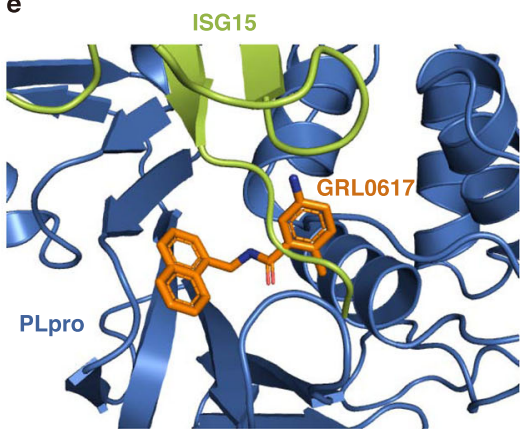

SARS-CoV-2-PLpro ${ }^{\text {c111s_-GRL0617 }}$ ISG15-PA-SRAS-CoV-2-PLpro (PDB:6XA9)

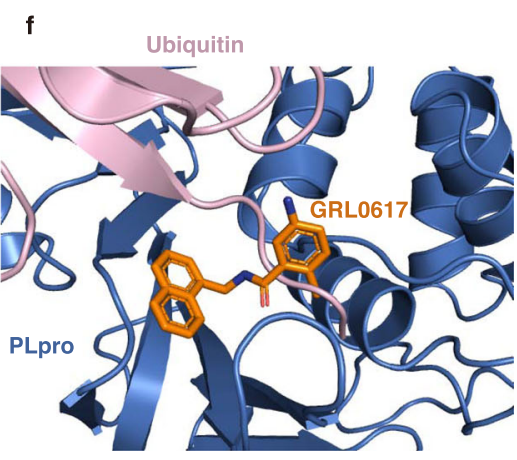

SARS-CoV-2-PLproc111s_GRL0617 Ub-PA-SARS-CoV-2-PLpro (PDB:6XAA)

Fig. 3 NMR studies show that GRL0617 blocks the binding of ISG15 to SARS-CoV-2 PLpro. a ${ }^{1} \mathrm{H}^{15} \mathrm{~N}-\mathrm{HSQC}$ spectrum of ${ }^{15} \mathrm{~N}-\mathrm{ISG} 15$. b HSQC spectrum of ${ }^{15} \mathrm{~N}-\mathrm{ISG} 15(0.1 \mathrm{mM})$ and $0.15 \mathrm{mM}$ PLpro. Peak broadening and peak intensity loss indicate binding of ISG15 to PLpro. c HSQC spectrum of $15 \mathrm{~N}-\mathrm{ISG} 15$ $(0.1 \mathrm{mM})$ in the mixture of $0.15 \mathrm{mM}$ PLpro and $0.25 \mathrm{mM}$ GRL0617. Recovery of peak intensity suggests that GRL0617 binds to PLpro and displaces ISG15. d SARS-CoV-2 PLproC111S/GRL0617 structure in cartoon model. e ISG15 in the complex structure of human ISG15 C-UBL-PA/SARS-CoV-2 PLpro (PDB 6XA9 [https://doi.org/10.2210/pdb6xa9/pdb]) was superimposed on (d) showing steric clash of GRL0617 with the C-terminal tail of ISG15. $\mathbf{f}$ Ub in the complex structure of UbPA/SARS-CoV-2 PLpro (PDB 6XAA [https://doi.org/10.2210/pdb6xaa/pdb]) was superimposed on (d), showing steric clash of GRL0617 with the C-terminal tail of Ub. 
added into the mix of $0.1 \mathrm{mM}{ }^{15} \mathrm{~N}-\mathrm{ISG} 15$ and $0.15 \mathrm{mM}$ SARSCoV-2-PLpro, a dose-dependent response of peak intensity recovery was evident, which suggested that GRL0617 competes with ISG15 for the binding site in PLpro, and blocks the binding of ISG15 to PLpro (Supplementary Fig. 6a). The superposition of the HSQC spectra of $0.1 \mathrm{mM}{ }^{15} \mathrm{~N}-\mathrm{ISG} 15$ only and the $0.1 \mathrm{mM}$ ${ }^{15} \mathrm{~N}$-ISG15/0.15 mM PLpro/0.25 mM GRL0617 mixture showed that these two spectra are essentially identical (Supplementary Fig. 6c), which indicated that GRL0617 is a potent binder to PLpro and almost completely abolished the binding of ISG15 to PLpro at a molar ratio of $1.67(0.25 \mathrm{mM} / 0.15 \mathrm{mM})$. No peak shifting was observed in the superimposed HSQC (Supplementary Fig. 6b), suggesting that GRL0617 is a bona fide binder of PLpro rather than ISG15 because the HSQC spectrum of ${ }^{15} \mathrm{~N}$ ISG15 is not disturbed at all by 2.5 excess molar ratio $(0.25 \mathrm{mM} /$ $0.10 \mathrm{mM}$ ) of GRL0617.

In comparison with the available complex structure of SARSCoV-2 PLpro with $\mathrm{Ub}^{32}$ (PDB ID: 6XAA [https://doi.org/ $10.2210 / \mathrm{pdb} 6 \mathrm{xaa} / \mathrm{pdb}$ ) (Fig. 3f) or ISG15 ${ }^{31,32}$ (PDB ID: 6XA9 [https://doi.org/10.2210/pdb6xa9/pdb] and 6YVA [https://doi. org/10.2210/pdb6yva/pdb]) (Fig. 3e), GRL0617 binds in the $\mathrm{S} 1$ site (for binding the C-terminal lobe of ISG15) and blocks the access of the C-terminal tail of proximal Ub or ISG15 to the active site of SARS-CoV-2 PLpro, respectively. Titrations of PLpro into ${ }^{15} \mathrm{~N}-\mathrm{Ub}$ caused minimal peak shifting or peak broadening even at a high molar ratio of 3 (Supplementary Fig. 7), showing much weaker binding for PLpro with monoUb compared with ISG15. Consequently, GRL0617 was not further titrated into the ${ }^{15} \mathrm{~N}-\mathrm{Ub} /$ PLpro mixture. Taken together, our NMR and X-ray analysis indicate that GRL0617 is a potent PPI inhibitor for PLpro by blocking the binding of ISG15 to PLpro.

The C-terminus of ISG15 is dominant for ISG15/SARS-CoV-2 PLpro binding. As seen in Supplementary Fig. 6c, superimposed NMR spectra indicated an almost complete disruption of interactions of the N- and C-UBL domains of ISG15 with PLpro. It is intriguing that GRL0617 actually only blocked the binding of the C-terminal tail of ISG15, but it efficiently abolished the binding of both the N- and C-globular UBL domains of ISG15 with PLpro. Therefore, GRL0617, as a small-molecule compound (MW = 304.3), occupies a cleft near the active site and exerts a dominant negative effect for the rest of ISG15 binding to SARS-CoV2 PLpro.

To further confirm the important role of the C-terminal tail of ISG15, we generated a truncated construct ISG15- $\Delta$ C6 (removing the C-terminal LRLRGG). As shown in the superimposed ${ }^{1} \mathrm{H},{ }^{15} \mathrm{~N}-\mathrm{HSQC}$ spectra of ${ }^{15} \mathrm{~N}-\mathrm{ISG} 15-\mathrm{FL}$ and ${ }^{15} \mathrm{~N}-\mathrm{ISG} 15-\Delta \mathrm{C} 6$ (Supplementary Fig. 8), ISG15- $\Delta$ C6 has a fold similar to the fulllength ISG15 (ISG15-FL). However, the superimposed ${ }^{1} \mathrm{H},{ }^{15} \mathrm{~N}$ HSQC also indicates that the interactions between ISG15 and SARS-CoV-2 PLpro were abolished by removal of the C-terminus of ISG15, as minimal peak shifting or peak broadening were observed for ${ }^{15} \mathrm{~N}-\mathrm{ISG} 15-\Delta \mathrm{C} 6 / \mathrm{SARS}-\mathrm{CoV}-2$ (molar ratio = 1:1.5) as compared with the massive peak broadening for ${ }^{15} \mathrm{~N}$-ISG15FL/SARS-CoV-2 (molar ratio $=1: 1.5) \quad$ (Fig. 4a, b). Isothermal titration calorimetry (ITC) experiments confirmed that removal of the C-terminus of ISG15 is detrimental to the binding of ISG15 and SARS-CoV-2 PLpro (Fig. 4c) (Table 1). Two complex structures of mouse full-length ISG15/SARS-CoV-2 PLpro (6VYA [https://doi.org/10.2210/pdb6yva/pdb]) and human ISG15 C-UBL-PA/SARS-CoV-2 PLpro (PDB 6XA9 [https://doi. org/10.2210/pdb6xa9/pdb]) were recently solved by the Dikic group $^{31}$ and the Komander group ${ }^{32}$, respectively. Further structural analysis of the human ISG15 C-UBL-PA/SARS-CoV2 PLpro reveals that the PLpro backbone of L162, G163, Y268,
C270, and G271 as well as sidechains of D164, R166, E167, and Y264 were involved in the interactions with the C-terminus of ISG15 through hydrogen bonds and electrostatic interactions. (Fig. 4d). Two mutants D164A and E167A were subsequently generated, and their enzymatic activities of cleaving Ub tags or ISG15 tags were examined using Ub-AMC or ISG15-AMC, respectively. Both D164A and E167A showed impaired activity on Ub and ISG15 cleaving (Fig. 4e). The cleavage of peptide substrate RLRGG-AMC also indicated diminished enzymatic activities for these mutants. These two mutants did not show observable activity on Ub-AMC at $1 \mu \mathrm{M}$ substrate concentration, presumably because SARS-CoV-2 PLpro cleaves Ub-AMC almost 10 -fold less efficiently than ISG15-AMC ${ }^{30}$.

We also examined the binding of ISG15- $\Delta$ C6 with SARS-CoV or MERS PLpro using ${ }^{1} \mathrm{H},{ }^{15} \mathrm{~N}$-HSQC spectra. Similar to SARSCoV-2 PLpro, the other two orthologs also showed minimum interactions (Supplementary Fig. 9a-c). ITC results confirmed lack of interactions between ISG15- $\triangle$ C6 and SARS-CoV-2, SARS$\mathrm{CoV}$, or MERS PLpro (Supplementary Fig. 9d-f). Another two truncated ISG15 mutants, i.e., ISG15- $\Delta$ C5 (removing C-terminal RLRGG) and ISG15- $\Delta$ C4 (removing C-terminal LRGG) were also generated and tested for their bindings with SARS-CoV-2 PLpro by ITC (Table 1 and Supplementary Fig. 9g, h). Similar to the shorter construct ISG15- $\Delta$ C6, ISG15- $\Delta$ C5 and ISG15- $\Delta$ C4 showed no observable binding with SARS-CoV-2 PLpro. These binding results suggest that the C-terminus of ISG15 is dominant for its binding with SARS-CoV-2 PLpro.

The extensive interaction network between ISG15 C-terminus and SARS-CoV-2 PLpro revealed by complex structures. Previous studies reveal that the C-terminal UBL domain of ISG15 is sufficient for binding with MERS PLpro ${ }^{38}$ or mammal USP $18^{39}$. Based on a complex structure of full-length ISG15 and MERS PLpro, it was suggested that C-terminal tail of ISG15 plays an important role in the hydrogen-bond network formed by ISG15 and MERS PLpro ${ }^{40}$. Other studies also suggested that the Cterminal RLRGG of Ub is responsible for a major part of interaction between $\mathrm{Ub}$ and SARS-CoV ${ }^{16}$, MERS PLpro ${ }^{16,41}$, or human DUBs ${ }^{42}$.

Accordingly, we analyzed available human C-UBL-ISG15-PA/ SARS-CoV-2 PLpro and mouse ISG15-FL/SARS-CoV-2 PLpro complex structures using the PISA (proteins, interfaces, structures, and assemblies) program ${ }^{43}$ (Fig. 5a, b and Supplementary Fig. 10). Comparing these two structures, it is clear that the majority of the interactions between ISG15 and PLpro is from the C-UBL domain of ISG15. In two recent studies ${ }^{31,32}$, the PLpro residues V66, F69, Y171, and N156 were reported for interacting with the $\mathrm{N}$ - or C-globular domains of ISG15 $5^{31,32}$. In addition, intermolecular interactions with the C-terminus of ISG15 involve PLpro residues G163, D164, E167, R166, Y264, Y268, and G271 (Fig. 5a, b and Supplementary Fig. 10). The backbone of G163, Y268, G271 and the sidechains of D164, R166, E167, Y264 formed hydrogen bonds and electrostatic interactions with PLpro (Fig. 5b and Supplementary Fig. 10c). By comparing the structures of apo- and ISG15-bound SARS-CoV-2 PLpro, a shift of the BL2 loop of PLpro was observed (Fig. 5c-e). In apoSARS-CoV-2 PLpro, the ISG15-C-terminus binding pocket takes an open conformation. Upon binding to ISG15, the PLpro BL2 loop shifts toward the substrate, i.e., the C-terminus of ISG15, and firmly holds the C-terminus through a hydrogenbond and electrostatic interaction network (Fig. 5). Using the sidechain $\mathrm{OH}$ of $\mathrm{Y} 268$ as a reference, the BL2 loop shifted $4.5 \AA$ to encircle the C-terminus of ISG15 (Fig. 5e), which also happened to the binding of GRL0617 in the same pocket (Fig. 2d). 
a
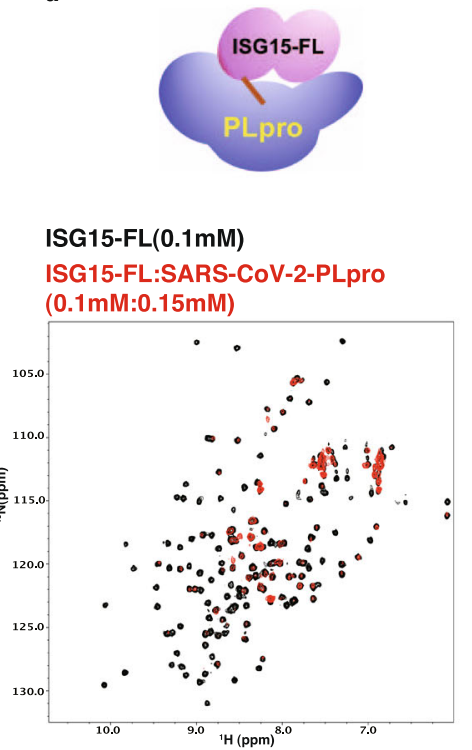

d

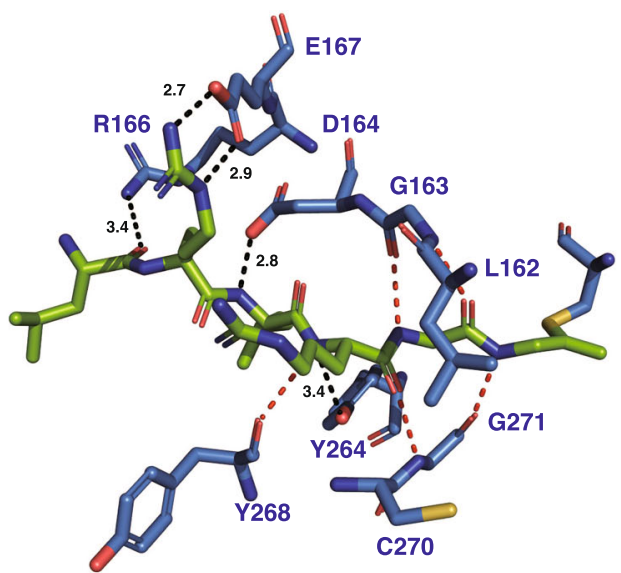

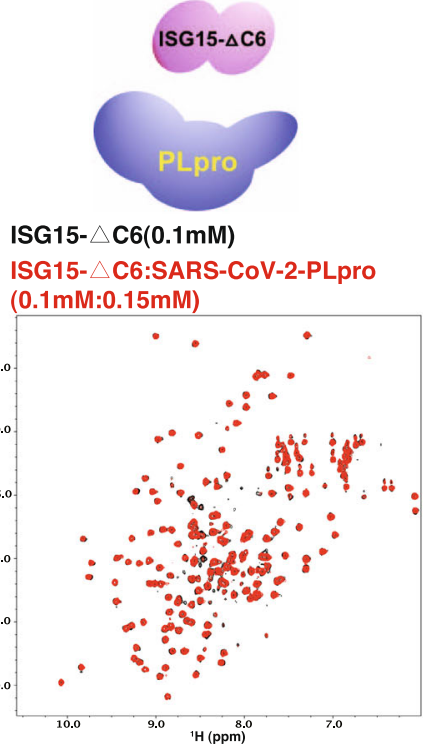
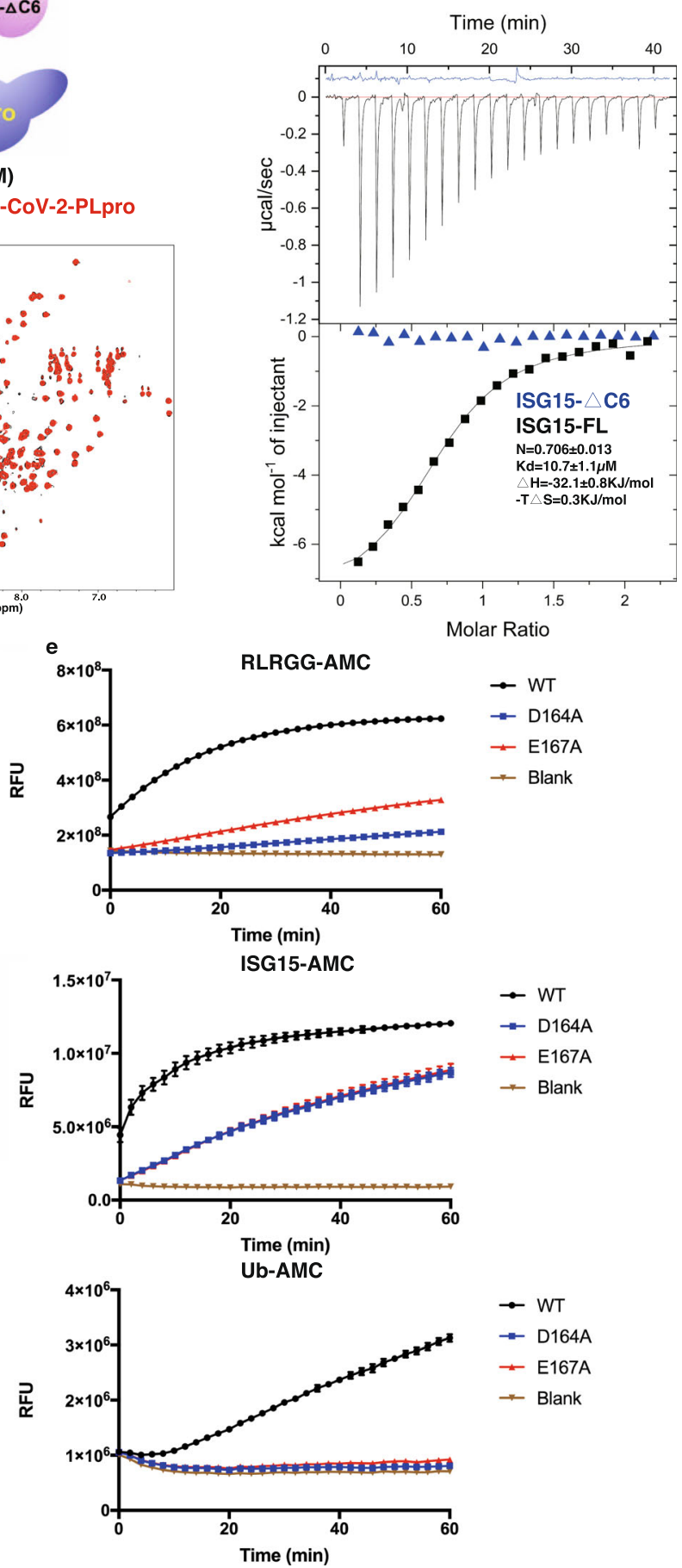

Fig. 4 The C-terminal tail of ISG15 is dominant for binding SARS-CoV-2 PLpro. a Superposition of the ${ }^{1} \mathrm{H}^{15} \mathrm{~N}-\mathrm{HSQC}$ spectra of ${ }^{15} \mathrm{~N}-\mathrm{ISG} 15-\mathrm{FL}(0.1 \mathrm{mM})$ and the mixture of ${ }^{15} \mathrm{~N}$-ISG15 $(0.1 \mathrm{mM}) / 0.15 \mathrm{mM}$ SARS-CoV-2 PLpro. Massive peak broadening and peak intensity loss indicate binding of ISG15-FL to PLpro. b Superposition of the ${ }^{1} \mathrm{H},{ }^{15} \mathrm{~N}-\mathrm{HSQC}$ spectra of ${ }^{15} \mathrm{~N}-\mathrm{ISG} 15-\Delta \mathrm{C} 6(0.1 \mathrm{mM})$ and the mixture of ${ }^{15} \mathrm{~N}-\mathrm{ISG} 15-\Delta \mathrm{C} 6(0.1 \mathrm{mM}) / \mathrm{SARS}-\mathrm{CoV}-2$ PLpro $(0.15 \mathrm{mM})$. Negligible peak perturbation indicates minimum binding of ISG15- $\Delta$ C6 to PLpro. c ITC measurement for the binding of SARS-CoV-2 with ISG15-FL (black) and ISG15- $\Delta$ C6 (blue), respectively. d Structural analysis of the complex structure of ISG15/SARS-CoV-2 PLpro (PDB 6XA9 [https:// doi.org/10.2210/pdb6xa9/pdb]) shows that the sidechains (black dashed lines) of D164 and E167 of SARS-CoV-2 PLpro are involved in the binding with the C-terminal tail of ISG15, other interactions are involved with the backbone (red dashed lines). e DUB cleavage assay using Ub-AMC, ISG15AMC, or peptide-AMC shows that D164A and E167A mutants have impaired enzyme activity compared with wild-type SARS-CoV-2 PLpro. Data are presented as mean $\pm \mathrm{SEM}, n=3$ independent experiments. 


\section{Discussion}

Our biochemical, structural, and antiviral data of SARS-CoV-2 PLpro support the claim that PLpro is a promising drug target for COVID-19 treatment. Our co-crystal structure of PLpro ${ }^{\text {Ci11S }}$ in complex with the potent inhibitor GRL0617 and its antiviral effect on Vero 6 cells validated that SARS-CoV-2 PLpro is a druggable target for SARS-CoV-2. In two recent studies, it was reported that the inhibition of deISGylating activity of PLpro is linked to the antiviral activity of PLpro inhibitors ${ }^{31,44}$. Our studies provided the mechanism of action for GRL0617. GRL0617 blocks the binding of ISG15 or Ub to PLpro, naturally it will also inhibit the processing of viral polyproteins of SARS-CoV-2 since these viral polyproteins share similar substrate cleavage site with Ub and ISG15.

The dominant role of the C-terminus of ISG15 in binding SARS-CoV-2 PLpro is intriguing. ISG15 contains $\mathrm{N}$ - and C-

\section{Table 1 The binding affinities of ISG15 and truncated mutants with SARS-CoV-2/SARS/MERS PLpro.}

\begin{tabular}{llll} 
& SARS-CoV-2-PLpro & SARS-PLpro & MERS-PLpro \\
\hline ISG15-FL & $10.7 \pm 1.1$ & $20.50 \pm 4.48^{64}$ & $59.3 \pm 12.7^{64}$ \\
ISG15- $\triangle$ C6 & N.D. ${ }^{*}$ & N.D. ${ }^{*}$ & N.D. ${ }^{\star}$ \\
ISG15- $\triangle$ C5 & N.D. & - & - \\
ISG15- $\triangle$ C4 & N.D. & - & - \\
\hline${ }^{*}$ N.D. & & & \\
\hline
\end{tabular}

terminal globular domains and a short C-terminal tail (residues RLRGG), which is often considered part of the C-domain ${ }^{45}$. Recent structural analysis reported that the SARS-CoV-2 PLpro S1 site is mainly for high ISG15 activity while the S2 site determines substrate selectivity 31,32 . In our study, we show that the short and linear C-terminal tail of ISG15 dominates its binding with PLpro. Because the tail is short and flexible, it offers transient and reversible interactions with its binding partner, i.e., PLpro in this case, which ensures optimal enzyme efficiency and high enzymatic turnover. Since the C-terminus of $\mathrm{Ub}$ is also heavily involved in its interactions with MERS or SARS-CoV PLpro ${ }^{16,41}$, primarily interacting with the C-terminus of Ub or ISG15 could be a general strategy for viral DUBs to attack host immune system.

The ISG15 C-terminus binding cleft in PLpro contributes a disproportionately large portion of the binding energy compared with the rest of the protein, therefore this is a hot spot pocket for antiviral PPI drug discovery. Small-molecule drugs often occupy hot spots on PPI interfaces and inhibit target proteins ${ }^{46}$. Because PLpro is a viral DUB with Ub and ISG15 cleavage functions, we compared our co-crystal structure with the structures of known USP7 and USP14 inhibitors to see if they share the same binding sites. Indeed, several non-covalent USP7 or USP14 inhibitors occupy the same pocket in the USP domain (Fig. 6) and block the binding of the C-terminus of $\mathrm{Ub}^{47-49}$. The binding site of USP7 inhibitors FT671 (PDB 5NGE [https://doi.org/10.2210/pdb5nge/ $\mathrm{pdb}])^{47}$, XL188 (PDB 5VS6 [https://doi.org/10.2210/pdb5vs6/ $\mathrm{pdb}])^{48}$, and ALM2 (PDB 5N9R [https://doi.org/10.2210/

a $\quad$ b

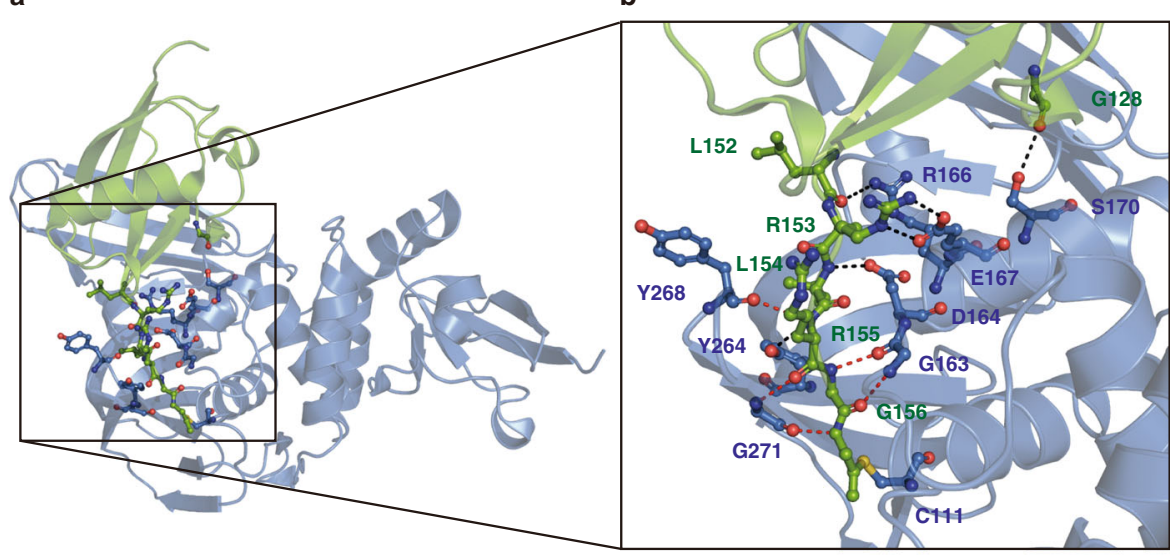

PDB: 6XA9

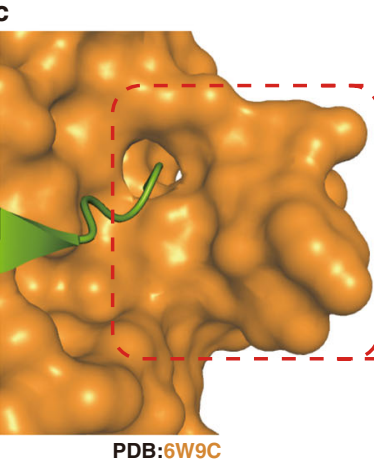

\section{d}

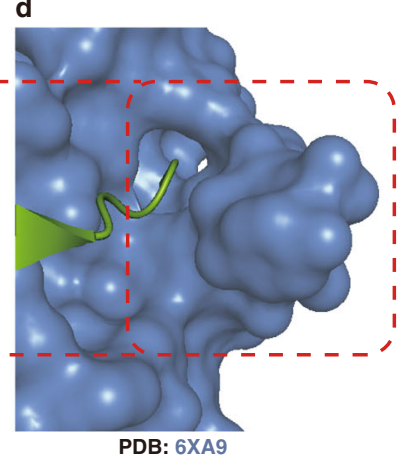

e

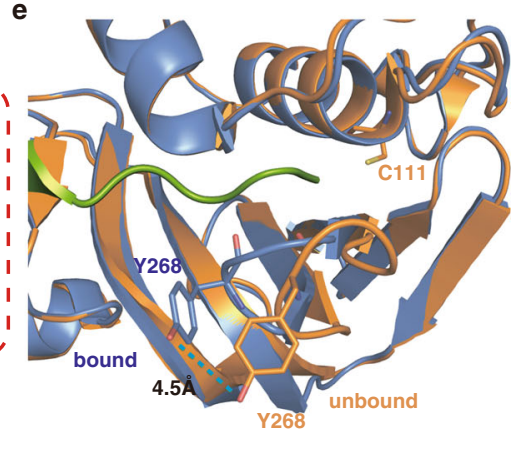

Fig. 5 The extensive hydrogen-bond and electrostatic interaction network between ISG15 C-terminus and SARS-CoV-2 PLpro. a The complex structure of human ISG15 C-UBL domain and SARS-CoV-2 PLpro (PDB 6XA9 [https://doi.org/10.2210/pdb6xa9/pdb]). b Close-up view for interactions of the Cterminus of ISG15 (green) with PLpro (marine), sidechain interactions in black dashed lines, and backbone interactions in red dashed lines. c Surface model showing the superposition of the C-terminus of ISG15 on apo-PLpro (PDB 6W9C [https://doi.org/10.2210/pdb6w9c/pdb]). d Surface model showing the C-terminus of ISG15 in PLpro (PDB 6XA9 [https://doi.org/10.2210/pdb6xa9/pdb]). e Cartoon model showing the shift of BL2 loop from the apo-state (orange) to bound state (marine), a distance of $4.5 \AA$ was labeled for the shifting of sidechain $\eta-O H$ of $Y 268$ in PLpro. 


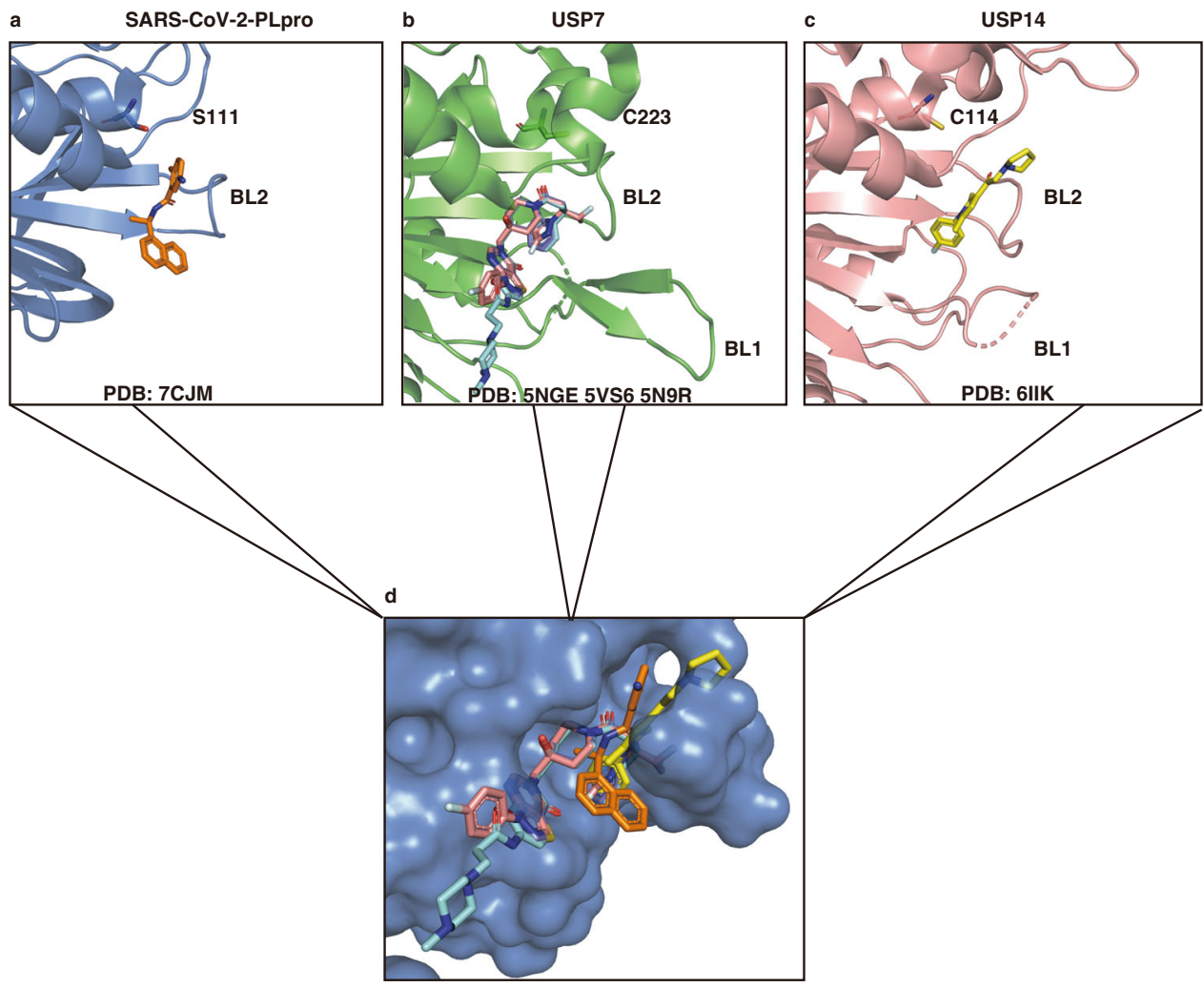

Fig. 6 GRL0617 occupies the same binding pocket in the USP domain as other USP7 and USP14 inhibitors. a Binding pocket of GRLO617 (orange) in SARS-CoV-2 PLproC111S mutant (PDB 7CJM [https://doi.org/10.2210/pdb7cjm/pdb]), the S111 was labeled in place of C111. b Binding pocket of FT671 (PDB 5NGE [https://doi.org/10.2210/pdb5gne/pdb], pink), XL188 (PDB 5VS6 [https://doi.org/10.2210/pdb5vs6/pdb], cyan), and ALM2 (PDB 5N9R [https://doi.org/10.2210/pdb5n9r/pdb], purple) in USP7 with active Cys223 label. c Binding pocket of IU1 (yellow) in USP14 (PDB 6IIK [https://doi.org/ 10.2210/pdb6iik/pdb]) with active Cys114 label; the BL1 and BL2 loops were labeled in (a, b, c). d Superposition of GRL0617, USP7, and USP14 in the ISG15 C-terminus binding pocket in SARS-CoV-2 PLpro.

pdb5n9r/pdb] $)^{49}$ largely overlap in the Ub-C-terminus binding cleft near the active Cys223; the USP14 inhibitor IU1 (PDB 6IIK [https://doi.org/10.2210/pdb6iik/pdb] $)^{50}$ and GRL0617 reside in the same pocket encircled by the BL2 loop (Fig. 6a, c). The superposition of all these DUB inhibitors on PLpro (Fig. 6d), shows that they are all at the same Ub or ISG15 C-terminus binding cleft, illustrating arguably the most important pocket in SARS-CoV-2 PLpro for the discovery of PPI inhibitors. In addition, analysis of available co-crystal structures of inhibitorbound DUBs suggests that stabilization of the inactive conformations of $\mathrm{DUB}^{51}$, and/or structural plasticity of the BL1/BL2 loops in DUB are also potential mechanisms of inhibition ${ }^{52}$. Since several independent high-throughput screening assays targeting SARS-CoV-2 using approved drugs have been unsuccessful ${ }^{32,53}$, structure-based drug discovery for this hot spot in PLpro and optimization of GRL0617 or its analogs with PLpro would be a promising approach for combating COVID-19. Recently reported covalent peptidic inhibitors targeting the active $\mathrm{C} 111$ also bind in this pocket in PLpro ${ }^{33}$.

Although the seven approved drugs obtained in our screening show low potency against PLpro, we cannot rule out the potentials of these drugs to therapeutically treat COVID-19 because they may have higher antiviral activities through other more complex mechanisms, e.g., the $6-\mathrm{TG}^{44}$.

In summary, we report the co-crystal structure of SARS-CoV-2 PLpro and GRL0617. We also found that the C-terminus of ISG15 is dominant in its binding with PLpro. The ISG15 Cterminus binding cleft in PLpro is a hot spot for non-covalent PPI inhibitor discovery. Our study implicates that it may be an efficient approach to focus on this pocket for future efforts of drug discovery targeting SARS-CoV-2 PLpro.

\section{Methods}

Plasmids construction, protein expression, and purification. Protein sequence for SARS-CoV-2 PLpro (amino acids, 746-1060) of Nsp3 protein from SARS-CoV2 (Nsp3; YP_009742610.1), protein sequence for SARS PLpro (amino acids, 7231037) of Nsp3 protein from SARS (Nsp3; NP_828862.2), and protein sequence for MERS PLpro (amino acids, 627-950) of Nsp3 protein from MERS(Nsp3;

YP_009047231.1) were codon-optimized (Supplementary Table 3), synthesized, and subcloned into pET28a vector with N-terminal His-tag and TEV protease site (General Biosystems, China). The C111S, D164A, and E167A mutations were introduced into the SARS-CoV-2-PLpro with a QuikChange site-directed mutagenesis kit (Agilent Technologies, USA) and primers (Supplementary Table 4). Protein sequence for Homo sapiens ISG15 (amino acids, 1-157) (ISG15; NP_005092.1) was synthesized and subcloned into pET28a with a tandem Nterminal His-tag, SUMO-tag, and TEV-protease site. ISG15 truncated mutants in the C-terminus $(\Delta \mathrm{C} 6, \Delta \mathrm{C} 5, \Delta \mathrm{C} 4)$ were constructed from above pET28a-SUMOISG15 plasmid by PCR and homologous recombination technology. Human Ub was codon-optimized, synthesized, and subcloned into pET28a with an $\mathrm{N}$-terminal His-tag and TEV-protease site. Protein sequence for Mus musculus USP18 (amino acids, 46-368) (USP18; CAJ18436.1P) was codon-optimized, synthesized, and subcloned into $\mathrm{pBac}$ vector with tandem N-terminal His-tag, SUMO-tag, and TEV protease site (Genewiz, China). All plasmids were verified by DNA sequencing analysis.

The plasmids were subsequently transformed into E. coli BL21 (DE3) cells. Protein expression was carried out in LB medium. E. coli cells were grown in LB medium at $37^{\circ} \mathrm{C}$ until $\mathrm{OD}_{600}$ reaches $0.8-1.0,0.5 \mathrm{mM}$ Isopropyl $\beta$-D-1-

thiogalactopyranoside (IPTG) and $100 \mathrm{mM} \mathrm{ZnSO}_{4}$ were added, and cells were growing overnight at $18^{\circ} \mathrm{C}$. For the production of ${ }^{15} \mathrm{~N}$-labeled ISG15 and Ub, protein samples were prepared by growing bacteria in M9 medium containing ${ }^{15} \mathrm{NH}_{4} \mathrm{Cl}$.

Cell pellets were resuspended in buffer A ( $30 \mathrm{mM}$ Tris, $400 \mathrm{mM} \mathrm{NaCl}, 30 \mathrm{mM}$ imidazole, $2 \mathrm{mM} \beta$-ME, pH 8.5) with the addition of $1 \mathrm{mM}$ phenylmethylsulfonyl 
fluoride (PMSF) and lysed using sonication. Cell lysate was subsequently centrifuged at $40,000 \mathrm{~g}$ at $4{ }^{\circ} \mathrm{C}$ for $1 \mathrm{~h}$. The supernatant was further loaded on a NiNTA column and then purified using buffer B $(30 \mathrm{mM}$ Tris, $400 \mathrm{mM} \mathrm{NaCl}, 300$ $\mathrm{mM}$ imidazole, $2 \mathrm{mM} \beta-\mathrm{Me}, \mathrm{pH}$ 8.5) on an AKTA Pure purification system (GE Healthcare). A second step of purification was carried out on a Superdex 200 gel filtration column using Buffer C (30 mM Tris, $100 \mathrm{mM} \mathrm{NaCl}, 1 \mathrm{mM}$ DTT, pH 8.5, for crystallization) or Buffer D (30 mM Tris, $100 \mathrm{mM} \mathrm{NaCl}, 1 \mathrm{mM} \mathrm{DTT,} \mathrm{pH} \mathrm{7.4,}$ for measuring the enzymatic activities and NMR tests). The fractions were pooled and concentrated to $10 \mathrm{mg} / \mathrm{mL}$ and stored at $-80^{\circ} \mathrm{C}$.

The mUSP18 was prepared with the MultiBac system 54,55 as described previously ${ }^{56}$. It is briefly described as follows: pBac-His-SUMO-TEV-mUSP18 was transformed into DH10EmBacY cells and the positive clones were screened and selected with a blue-white screening protocol. The virus bacmid was transfected into Sf9 cells (Thermo Fisher Scientific) with lipofectamine 2000 (Thermo Fisher Scientific). Sf 9 cells were grown in Sf-900 ${ }^{\text {tox }}$ II SFM media (Thermo Fisher Scientific). The initial virus was harvested and successful infection was monitored by the measurement of yellow fluorescent protein expression using a fluorescence spectrophotometer. Western blotting was used to test the expression of mUSP18. For large-scale expression of mUSP18, $100 \mathrm{~mL}$ of Sf9 cells at a density of $1 \times 10^{6}$ cells $/ \mathrm{mL}$ were infected with $200-600 \mu \mathrm{L}$ of the virus. The cells were kept at the same density until proliferation arrest. Then the cells were harvested for purification. The purification process is the same as for PLpro proteins.

Cell culture and plasmid transfection. HEK293T(ATCC) and Vero E6 (Shanghai Institutes for Biological Sciences, Chinese Academy of Sciences) cells were cultured in DMEM (Dulbecco's Modified Eagle Medium; Gibco) medium supplemented with $10 \%$ (vol/vol) FBS (Fetal Bovine Serum; Gibco) and penicillin $(100 \mathrm{U} / \mathrm{mL}) /$ streptomycin $(100 \mu \mathrm{g} / \mathrm{mL})$. In transient-transfection experiments, cells at $70 \%$ confluency were transfected with plasmid DNA constructs using Lipofectamine 3000 reagents (Invitrogen, L3000015).

Immunoblotting for detection of ISGylation. HEK293T cells were co-transfected with plasmids encoding Myc-tagged ISG15, pcDNA3,1-UBE1L (E1), UBCH8 (E2), or 3XFlag-HERC5 (E3) for $24 \mathrm{~h}$. Cell extracts were prepared in RIPA lysis buffer (20 mM Tris-HCl (pH 7.5), $150 \mathrm{mM} \mathrm{NaCl}, 1 \mathrm{mM}$ EDTA, $1 \% \mathrm{NP}-40,1 \%$ SDS, and protease inhibitor cocktail (Roche Diagnostics, Germany, 5892791001), pH 7.5. Protein concentrations were determined by Bradford assay (Bio-Rad Laboratories, USA, 500-0205). Equal amounts proteins $(40 \mu \mathrm{g})$ were subjected to SDS-PAGE, followed by transferring to nitrocellulose membranes. Membranes were blocked with TBST containing 5\% skim-milk for $1 \mathrm{~h}$ at room temperature and then overnight with ISG15 (1:1000, Thermo Fisher Scientific, USA, 703131), GFP (1:1000, Abclonal, China, AE012), GAPDH (1:5000, Abclonal, China, AC002), and Ubiquitin (1:1000, Cell Signal Technology, USA, \#3936) antibodies at $4^{\circ} \mathrm{C}$. After three washing steps, membranes were incubated with HRP-conjugated secondary antibodies (1:10000, Santa Cruz Biotechnology, TX, sc-516102, 1:10000, Enzo, ADI-SAB-300) for $1 \mathrm{~h}$ at room temperature and visualized using ChemiDoc system (Bio-Rad Laboratories, USA).

In the assay of IFN- $\beta$ (Sino Biological Inc., 10704-HNAS) induced ISGylation and the inhibition of PLpro by GRL0617, HEK293T cells were first treated with $500 \mathrm{U} / \mathrm{mL}$ IFN- $\beta$ for $48 \mathrm{~h}$ and cell extracts were prepared in RIPA lysis buffer; $30 \mu \mathrm{g}$ of extracts were mixed with purified recombinant PLpro $(100 \mathrm{nM})$ and indicated concentrations of GRL0617 in the reaction buffer $(50 \mathrm{mM}$ Tris- $\mathrm{HCl}, 50 \mathrm{mM} \mathrm{NaCl}$, $5 \mathrm{mM}$ DTT, $\mathrm{pH} 7.5$ ). Reactions were incubated at $37^{\circ} \mathrm{C}$ for $1 \mathrm{~h}$ and prepared for immunoblotting analysis as indicated.

PLpro activity assays and $\mathbf{I C}_{\mathbf{5 0}}$ determination. In this study, PLpro activity was monitored using the substrate peptide-AMC (Z-Arg-Leu-Arg-Gly-Gly-AMC, Cat. No. 4027158, Bachem Bioscience). Experiments were performed in 384-well black non-binding plates (Cat. No. 3575, Corning) with a final reaction volume of $50 \mu \mathrm{L}$. The assay buffer contained $50 \mathrm{mM}$ HEPES, $\mathrm{pH} 7.4,0.01 \%$ Triton X-100 (v/v), $0.1 \mathrm{mg} / \mathrm{mL} \mathrm{BSA}$, and $2 \mathrm{mM}$ DTT. PLpro was added to the plates at a final concentration of $100 \mathrm{nM}$. Enzyme reactions were initiated with $5 \mu \mathrm{L}$ of peptide-AMC (final $50 \mu \mathrm{M}$ ) dissolved in the above assay buffer. Upon addition of peptide substrate, the fluorescence signals were monitored at $340 \mathrm{~nm}$ (excitation) and $450 \mathrm{~nm}$ (emission) with 3 min intervals in a 2104 EnVision Multilabel Plate Reader (PerkinElmer)

An approved drug library (TargetMol, USA), of 2040 compounds including drugs approved by US FDA and CFDA, was used. The first round screening reaction mixture included $100 \mathrm{nM}$ PLpro, $50 \mu \mathrm{M}$ substrate, and $100 \mu \mathrm{M}$ compounds. The top $1.5 \%$ compounds of each plate were selected with a minimum $50 \%$ inhibition using the reaction in DMSO as a control. Around 30 compounds were tested in the second round of screening using the same assay and 23 compounds were excluded due to their reactivities, insolubility, or fluorescence interference. To determine the $\mathrm{IC}_{50}$ values of the remaining 7 compounds, a series of 8-point, $1: 2$ serial dilutions was performed from a highest starting concentration of $200 \mu \mathrm{M}$. Seven drugs used in this study were purchased from TargetMol (USA). The data were fitted using GraphPad Prism.
To test whether GRL0617 can inhibit mouse deISGylating enzyme USP18, its activity was assayed in the presence of an inhibitor with $250 \mathrm{nM}$ ISG15-AMC (Boston Biochem) as substrate (excitation: $340 \mathrm{~nm}$; emission: $450 \mathrm{~nm}$ ).

The activities of wild-type PLpro, D164A, and E167A (all at $100 \mathrm{nM}$ ) were tested on peptide-AMC $(100 \mu \mathrm{M})$, Ub-AMC $(1 \mu \mathrm{M}$, Boston Biochem), or ISG15$\mathrm{AMC}(1 \mu \mathrm{M})$ and were determined and compared.

Crystallization and data collection. The complex of GRL0617 (Cat. No. HY$117043, \mathrm{MCE}$ ) with PLpro ${ }^{\mathrm{C} 111 \mathrm{~S}}$ was crystallized by vapor diffusion in a sitting-drop format after a $20 \mathrm{~h}$ incubation of $9.5 \mathrm{mg} / \mathrm{mL}$ PLpro in the buffer $(50 \mathrm{mM}$ Tris, $\mathrm{pH}$ $8.5,100 \mathrm{mM} \mathrm{NaCl}$ ) with $2 \mathrm{mM}$ inhibitor at $4{ }^{\circ} \mathrm{C}$. Immediately before crystallization, the sample was clarified by centrifugation. A $0.75 \mu \mathrm{L}$ volume of the enzymeinhibitor solution was then mixed with an equal volume of well solution containing $5 \mathrm{mM}$ Cobalt (II) chloride hexahydrate; $5 \mathrm{mM}$ Cadmium chloride hemi (pentahydrate); $5 \mathrm{mM}$ Magnesium chloride hexahydrate; $5 \mathrm{mM}$ Nickel (II) chloride hexahydrate; $0.1 \mathrm{M}$ HEPES, $\mathrm{pH} 7.5$; and 12\% PEG 3350 and equilibrated against well solution at $12{ }^{\circ} \mathrm{C}$. Before data collection, crystals were soaked in a cryo solution containing well solution, $400 \mu \mathrm{M}$ inhibitor, and $20 \%$ glycerol. Crystals were flashfrozen in liquid $\mathrm{N}_{2}$. All diffraction data were collected at an in-house light source Rigaku MicroMax-007 HF and indexed, integrated, and scaled using CrysAlisPro and $\mathrm{XDS}^{57}$

Structure determination and data deposition. Structures were solved by molecular replacement using the apo-PLpro ${ }^{\mathrm{C} 111 \mathrm{~S}}$ structure (PDB 6WRH [https://doi. org $/ 10.2210 / \mathrm{pdb} 6 \mathrm{wrh} / \mathrm{pdb}])$ as the template. The MOLREP ${ }^{58}$ in CCP ${ }^{59}$ was used for MR. Structures were refined in PHINEX ${ }^{60}$ with manual model building in $\operatorname{Coot}^{61}$. Detailed statistics on the data collection and the final models for crystallographic analysis are shown in Supplementary Table 2. Structural models were assessed using MolProbity ${ }^{62}$. Structural alignments and graphical representations were generated using PyMOL ${ }^{63}$.

\section{Isothermal titration calorimetry of ISG15 and its truncated mutants with} PLpro proteins. ITC was performed using a Microcal-ITC200. One injection with $0.4 \mu \mathrm{L}$ and subsequent 19 injections of $2 \mu \mathrm{L}$ were performed at $25^{\circ} \mathrm{C}$ with a reference power of $5 \mu \mathrm{cal} / \mathrm{s}$. The ISG15 and its truncated mutants with SARS-CoV2/SARS-CoV/MERS PLpro proteins were all in PBS buffer. For binding experiment of SARS-CoV-2 PLpro with ISG15 or ISG15- $\triangle \mathrm{C} 6,92 \mu \mathrm{M}$ PLpro was placed in the cell with $0.95 \mathrm{mM}$ of ISG15 or $1 \mathrm{mM}$ ISG15- $\triangle \mathrm{C} 6$ in the syringe. For binding experiment of SARS-CoV or MERS PLpro with ISG15- $\triangle \mathrm{C} 6,100 \mu \mathrm{M}$ of SARS $\mathrm{CoV}$ or MERS PLpro was placed in the cell with $1.2 \mathrm{mM}$ or $1 \mathrm{mM}$ ISG15- $\triangle \mathrm{C} 6$ in the syringe. For binding experiment of SARS-CoV-2 PLpro with ISG15 truncated mutants $(\triangle \mathrm{C} 5, \triangle \mathrm{C} 4), 100 \mu \mathrm{M}$ of SARS-CoV-2 PLpro was placed in cell with 1-2 $\mathrm{mM}$ various ISG15 mutants in the syringe. The data were processed using Microcal-ITC200 analysis Software.

Antiviral and cytotoxicity assay. For the assay, $1 \times 10^{4}$ Vero E6 cells were seeded in triplicates in 96-well plates. After 20-24 h, fresh medium with different concentrations $(100,50,25,12.5,6.25,3.125$, and $1.56 \mu \mathrm{M})$ of inhibitors, DMSO as a control, was replaced. For measuring the cytotoxicity, cells were incubated for $48 \mathrm{~h}$ followed by the cell viability test with CCK8 reagent. For measuring the antiviral activity, cells were kept in the medium with inhibitors for $1 \mathrm{~h}$, followed by infection with SARS-CoV-2 virus strain BetaCoV/Shenzhen/SZTH-003/2020, which was clinically isolated from local patients, at $\mathrm{MOI}=0.01$. After a $2 \mathrm{~h}$ incubation, the virus-compound mixture was subsequently removed, and fresh medium containing candidate compounds $(100,50,25,12.5,6.25,3.125$, and $1.56 \mu \mathrm{M})$ or DMSO was added, and cell growth was continued for $48 \mathrm{~h}$. The viral RNA was extracted from the supernatant medium and the qRT-PCR assay was performed. The linearized plasmid containing the Spike gene of the SARS-CoV-2 virus was transcribed in vitro and was used to prepare a standard curve to quantify the copy number of the virus as previously described ${ }^{11}$. Briefly, the SARS-CoV-2 Spike gene were synthetized in pcDNA3.1 (Sangon, China). This vector was then linearized by single locus restriction endonuclease and subjected to in vitro transcription. The concentration of resulting RNA transcripts was measured by NanoDrop 2000. Microgram concentration of the plasmid can be transformed to the RNA copies. The first dilution is $10^{7}$ copies, then diluted to $10^{2}$ copies ( 6 diluted concentrations: $\left.10^{7}, 10^{6}, 10^{5}, 10^{4}, 10^{3}, 10^{2}\right)$. The qRT-PCR assay was performed with the above template and the probe, and primers of Spike gene. Standard curves were prepared according to the results of PCR amplification. The standard curve formula is $y=$ $-3.2998 \mathrm{x}+37.758, R^{2}=0.9997$. Primer and probe information: TaqMan primers for COVID-19 virus: $5^{\prime}$ TCCTGGTGATTCTTCTTCAGG-3' and $5^{\prime}$-TCTGAGA GAGGGTCAAGTGC- $3^{\prime}$ and COVID-19 virus probe $5^{\prime}$-FAM-AGCTGCAGCAC CAGCTGTCCA-BHQ1-3'. Data analysis was done with GraphPad Prism.

SARS-CoV-2-induced CPE on Vero E6 cells was observed and analyzed using reverse-phase light microscope. The inhibition effect of GRL0617 against SARS$\mathrm{CoV}-2$-induced cytopathogenic effect was measured under different concentrations. The cytopathic effect was examined for 3 days post-infection. The complete absence of cytopathic effect in an individual culture well was defined as protection. The value of $\mathrm{EC}_{50}$ was calculated using GraphPad prism software. 
NMR spectroscopy. NMR data were acquired at $25^{\circ} \mathrm{C}$ on a $600 \mathrm{MHz}$ Bruker AVANCE III spectrometer. The $600 \mathrm{MHz}$ spectrometer was equipped with a $5 \mathrm{~mm}$ TCI Cryoprobe. In NMR titrations, the samples of $0.1 \mathrm{mM}{ }^{15} \mathrm{~N}$-labeled ISG15 were incubated in the presence or absence of $0.15 \mathrm{mM}$ PLpro with or without the indicated concentration of GRL0617 $(0.05 \mathrm{mM}, 0.15 \mathrm{mM}$, and $0.25 \mathrm{mM})$ were investigated in assay buffer containing $30 \mathrm{mM}$ Tris, $100 \mathrm{mM} \mathrm{NaCl}, \mathrm{pH} \mathrm{7.4,5 \%}$ DMSO, and $10 \% \mathrm{D}_{2} \mathrm{O}$. For Ub titrations, the samples of $0.1 \mathrm{mM}^{15} \mathrm{~N}$-labeled $\mathrm{Ub}$ were incubated with PLpro $(0.1 \mathrm{mM}, 0.2 \mathrm{mM}$, and $0.3 \mathrm{mM}) .{ }^{1} \mathrm{H},{ }^{15} \mathrm{~N}-\mathrm{HSQC}$ titration spectra were collected for all the samples. All of the NMR spectra were processed using NMRPipe/NMRDraw and further analyzed using NMRView.

Reporting summary. Further information on research design is available in the Nature Research Reporting Summary linked to this article.

\section{Data availability}

Coordinates and structure factors were deposited in the PDB under accession code 7CJM (GRL0617-bound PLproC111S). Protein sequence for SARS-CoV-2 PLpro (amino acids, 746-1060) of Nsp3 protein from SARS-CoV-2 (Nsp3; YP_009742610.1). Protein sequence for SARS PLpro (amino acids, 723-1037) of Nsp3 protein from SARS (Nsp3; NP_828862.2). Protein sequence for MERS PLpro (amino acids, 627-950) of Nsp3 protein from MERS (Nsp3; YP_009047231.1). Protein sequence for Homo sapiens ISG15 (amino acids, 1-157) (ISG15; NP_005092.1). Protein sequence for Mus musculus USP18 (amino acids, 46-368) (USP18; CAJ18436.1 P). All relevant data are available upon request from the corresponding authors. Source data are provided with this paper.

Received: 31 July 2020; Accepted: 16 December 2020;

Published online: 20 January 2021

\section{References}

1. World Health Organization. Coronavirus Disease (COVID-2019) Situation Reports-176 https://www.who.int/emergencies/diseases/novel-coronavirus2019/situation-reports (WHO, 2020).

2. Zhou, P. et al. A pneumonia outbreak associated with a new coronavirus of probable bat origin. Nature 579, 270-273 (2020).

3. $\mathrm{Wu}, \mathrm{F}$. et al. A new coronavirus associated with human respiratory disease in China. Nature 579, 265-269 (2020).

4. Lu, R. et al. Genomic characterisation and epidemiology of 2019 novel coronavirus: implications for virus origins and receptor binding. Lancet 395, 565-574 (2020).

5. Chan, J. F. et al. A familial cluster of pneumonia associated with the 2019 novel coronavirus indicating person-to-person transmission: a study of a family cluster. Lancet 395, 514-523 (2020).

6. Zhu, N. et al. A novel coronavirus from patients with pneumonia in China, 2019. N. Engl. J. Med. 382, 727-733 (2020).

7. Wu, D., Wu, T., Liu, Q. \& Yang, Z. The SARS-CoV-2 outbreak: what we know. Int. J. Infect. Dis. 94, 44-48 (2020).

8. Yuen, K.S., Ye, Z.W., Fung, S.Y., Chan, C.P. \& Jin, D.Y. SARS-CoV-2 and COVID-19: the most important research questions. Cell Biosci. 10, 40 (2020).

9. Petrovski, B. E. et al. Reorganize and survive-a recommendation for healthcare services affected by COVID-19-the ophthalmology experience. Eye (Lond.) 34, 1177-1179 (2020).

10. Consortium, W.H.O.S.T. et al. Repurposed Antiviral Drugs for Covid-19 Interim WHO Solidarity Trial Results. N. Engl. J. Med. https://doi.org/ 10.1056/NEJMoa2023184 (2020).

11. Jin, Z. et al. Structure of $\mathrm{M}$ (pro) from SARS-CoV-2 and discovery of its inhibitors. Nature 582, 289-293 (2020).

12. Dai, W. et al. Structure-based design of antiviral drug candidates targeting the SARS-CoV-2 main protease. Science 368, 1331-1335 (2020).

13. Jin, Z. et al. Structural basis for the inhibition of SARS-CoV-2 main protease by antineoplastic drug carmofur. Nat. Struct. Mol. Biol. 27, 529-532 (2020).

14. Ma, C. et al. Boceprevir, GC-376, and calpain inhibitors II, XII inhibit SARS$\mathrm{CoV}-2$ viral replication by targeting the viral main protease. Cell Res. 30, 678-692 (2020).

15. Baez-Santos, Y. M., St John, S. E. \& Mesecar, A. D. The SARS-coronavirus papain-like protease: structure, function and inhibition by designed antiviral compounds. Antivir. Res. 115, 21-38 (2015).

16. Ratia, K., Kilianski, A., Baez-Santos, Y. M., Baker, S. C. \& Mesecar, A. Structural basis for the ubiquitin-linkage specificity and deISGylating activity of SARS-CoV papain-like protease. PLoS Pathog. 10, e1004113 (2014).

17. Clementz, M. A. et al. Deubiquitinating and interferon antagonism activities of coronavirus papain-like proteases. J. Virol. 84, 4619-4629 (2010).

18. Perng, Y. C. \& Lenschow, D. J. ISG15 in antiviral immunity and beyond. Nat. Rev. Microbiol. 16, 423-439 (2018).
19. Morales, D. J. \& Lenschow, D. J. The antiviral activities of ISG15. J. Mol. Biol. 425, 4995-5008 (2013).

20. Hermann, M. \& Bogunovic, D. ISG15: in sickness and in health. Trends Immunol. 38, 79-93 (2017).

21. Swaim, C. D. et al. Modulation of extracellular ISG15 signaling by pathogens and viral effector proteins. Cell Rep. 31, 107772 (2020).

22. Liu, C. et al. Research and development on therapeutic agents and vaccines for COVID-19 and related human coronavirus diseases. ACS Cent. Sci. 6, 315-331 (2020).

23. Li, G. \& De Clercq, E. Therapeutic options for the 2019 novel coronavirus (2019-nCoV). Nat. Rev. Drug Discov. 19, 149-150 (2020).

24. Guy, R. K., DiPaola, R. S., Romanelli, F. \& Dutch, R. E. Rapid repurposing of drugs for COVID-19. Science 368, 829-830 (2020).

25. Anson, B. J. et al. Broad-spectrum inhibition of coronavirus main and papainlike proteases by HCV drugs. Preprint at https://doi.org/10.21203/rs.3.rs26344/v1 (2020)

26. Ratia, K. et al. A noncovalent class of papain-like protease/deubiquitinase inhibitors blocks SARS virus replication. Proc. Natl Acad. Sci. USA 105, 16119-16124 (2008).

27. Chou, C. Y. et al. Thiopurine analogues inhibit papain-like protease of severe acute respiratory syndrome coronavirus. Biochem. Pharmacol. 75, 1601-1609 (2008).

28. Cheng, K. W. et al. Thiopurine analogs and mycophenolic acid synergistically inhibit the papain-like protease of Middle East respiratory syndrome coronavirus. Antivir. Res. 115, 9-16 (2015).

29. Ghosh, A. K. et al. Severe acute respiratory syndrome coronavirus papain-like novel protease inhibitors: design, synthesis, protein-ligand X-ray structure and biological evaluation. J. Med. Chem. 53, 4968-4979 (2010).

30. Freitas, B. T. et al. Characterization and noncovalent inhibition of the deubiquitinase and delSGylase activity of SARS-CoV-2 papain-like protease. ACS Infect. Dis. 6, 2099-2109 (2020).

31. Shin, D. et al. Papain-like protease regulates SARS-CoV-2 viral spread and innate immunity. Nature 587, 657-662 (2020).

32. Klemm, T. et al. Mechanism and inhibition of the papain-like protease, PLpro, of SARS-CoV-2. EMBO J. 39, e106275 (2020).

33. Rut, W. et al. Activity profiling and crystal structures of inhibitor-bound SARS-CoV-2 papain-like protease: a framework for anti-COVID-19 drug design. Sci. Adv. 6, eabd4596 (2020).

34. Osipiuk, J. et al. Structure of papain-like protease from SARS-CoV-2 and its complexes with non-covalent inhibitors. Preprint at https://doi.org/10.1101/ 2020.08.06.240192 (2020).

35. Gao, X. et al. Crystal structure of SARS-CoV-2 papain-like protease. Acta Pharm. Sin. B https://doi.org/10.1016/j.apsb.2020.08.014 (2020).

36. Baez-Santos, Y. M., Mielech, A. M., Deng, X., Baker, S. \& Mesecar, A. D. Catalytic function and substrate specificity of the papain-like protease domain of nsp3 from the Middle East respiratory syndrome coronavirus. J. Virol. 88, 12511-12527 (2014).

37. Lee, H. et al. Inhibitor recognition specificity of MERS-CoV papain-like protease may differ from that of SARS-CoV. ACS Chem. Biol. 10, 1456-1465 (2015).

38. Daczkowski, C.M., Goodwin, O.Y., Dzimianski, J.V., Farhat, J.J. \& Pegan, S.D. Structurally guided removal of DeISGylase biochemical activity from papainlike protease originating from Middle East respiratory syndrome coronavirus. J. Virol. 91, e01067-17 (2017).

39. Basters, A. et al. Structural basis of the specificity of USP18 toward ISG15. Nat Struct. Mol. Biol. 24, 270-278 (2017).

40. Clasman, J. R., Everett, R. K., Srinivasan, K. \& Mesecar, A. D. Decoupling deISGylating and deubiquitinating activities of the MERS virus papain-like protease. Antivir. Res. 174, 104661 (2020).

41. Lei, J. \& Hilgenfeld, R. Structural and mutational analysis of the interaction between the Middle-East respiratory syndrome coronavirus (MERS-CoV) papain-like protease and human ubiquitin. Virologica Sin. 31, 288-299 (2016).

42. Mevissen, T. E. T. \& Komander, D. Mechanisms of deubiquitinase specificity and regulation. Annu. Rev. Biochem. 86, 159-192 (2017).

43. Krissinel, E. \& Henrick, K. Inference of macromolecular assemblies from crystalline state. J. Mol. Biol. 372, 774-797 (2007).

44. Swaim, C. D. et al. 6-Thioguanine blocks SARS-CoV-2 replication by inhibition of PLpro protease activities. Preprint at https://doi.org/10.1101/ 2020.07.01.183020 (2020).

45. Narasimhan, J. et al. Crystal structure of the interferon-induced ubiquitin-like protein ISG15. J. Biol. Chem. 280, 27356-27365 (2005).

46. Cukuroglu, E., Engin, H. B., Gursoy, A. \& Keskin, O. Hot spots in proteinprotein interfaces: towards drug discovery. Prog. Biophys. Mol. Biol. 116, 165-173 (2014).

47. Turnbull, A. P. et al. Molecular basis of USP7 inhibition by selective smallmolecule inhibitors. Nature 550, 481-486 (2017). 
48. Lamberto, I. et al. Structure-guided development of a potent and selective non-covalent active-site inhibitor of USP7. Cell Chem. Biol. 24, 1490-1500 e11 (2017).

49. Gavory, G. et al. Discovery and characterization of highly potent and selective allosteric USP7 inhibitors. Nat. Chem. Biol. 14, 118-125 (2018).

50. Wang, Y. et al. Small molecule inhibitors reveal allosteric regulation of USP14 via steric blockade. Cell Res. 28, 1186-1194 (2018)

51. Wertz, I. E. \& Murray, J. M. Structurally-defined deubiquitinase inhibitors provide opportunities to investigate disease mechanisms. Drug Discov. Today Technol. 31, 109-123 (2019).

52. Schauer, N. J., Magin, R. S., Liu, X., Doherty, L. M. \& Buhrlage, S. J. Advances in discovering deubiquitinating enzyme (DUB) inhibitors. J. Med. Chem. 63, 2731-2750 (2020).

53. Smith, E. et al. High-Throughput Screening for Drugs That Inhibit PapainLike Protease in SARS-CoV-2. SLAS Discov. 25, 1152-1161 (2020).

54. Bieniossek, C., Richmond, T. J. \& Berger, I. MultiBac: multigene baculovirusbased eukaryotic protein complex production. Curr. Protoc. Protein Sci. 5, Unit 520 (2008)

55. Berger, I., Fitzgerald, D. J. \& Richmond, T. J. Baculovirus expression system for heterologous multiprotein complexes. Nat. Biotechnol. 22, 1583-1587 (2004).

56. Basters, A. et al. Molecular characterization of ubiquitin-specific protease 18 reveals substrate specificity for interferon-stimulated gene 15. FEBS J. 281, 1918-1928 (2014).

57. Kabsch, W. Xds. Acta Crystallogr. D. 66, 125-132 (2010).

58. Navaza, J. \& Saludjian, P. [33] AMoRe: an automated molecular replacement program package. Methods Enzymol. 276, 581-594 (1997).

59. Winn, M. D. et al. Overview of the CCP4 suite and current developments. Acta Crystallogr. D 67, 235-242 (2011).

60. Liebschner, D. et al. Macromolecular structure determination using X-rays, neutrons and electrons: recent developments in Phenix. Acta Crystallogr. D 75, 861-877 (2019).

61. Emsley, P. \& Cowtan, K. Coot: model-building tools for molecular graphics. Acta Crystallogr. D 60, 2126-2132 (2004).

62. Chen, V. B. et al. MolProbity: all-atom structure validation for macromolecular crystallography. Acta Crystallogr. D 66, 12-21 (2010).

63. DeLano, W. L. Use of PYMOL as a communications tool for molecular science. Abstr. Papers Am. Chem. Soc. 228, U313-U314 (2004).

64. Daczkowski, C. M. et al. Structural insights into the interaction of coronavirus papain-like proteases and interferon-stimulated gene product 15 from different species. J. Mol. Biol. 429, 1661-1683 (2017)

\section{Acknowledgements}

We thank Dr. Hans J. Vogel (University of Calgary, Canada) for critical comments and editing the manuscript. We also thank Dr. Jiali Gao and Dr. Feng Sha (Shenzhen Bay Laboratory, China) for helpful discussions, and Mr. Yunnan Hou (Peking University Shenzhen Graduate School) for technical assistance. All NMR experiments were performed at the Beijing NMR Center and the NMR facility of National Center for Protein Sciences at Peking University. Research reported in this publication was supported in whole or in part by the National Natural Science Foundation of China (grant no: 21977009 to H.H.), the Shenzhen Science and Technology Innovation Committee (grant no: JCYJ20170412150913708 to H.H.), the Shenzhen Bay Laboratory Open Research Program (grant nos: SZBL2019062801010 to H.H. and SZBL202002271003 to G.Z.), the Guangdong Scientific and Technological Project (2020B1111340076 to G.Z.) and the Natural Science Foundation of the Guangdong Province (Grant No: 2018A0303130091 to Z.F.).

\section{Author contributions}

H.H. and G.Z. conceived the project. H.H., Z.F., J.T., Y.X., W.Z., J.L. and Z.L. performed cloning, protein expression, isotope labeling and protein purification. Z.F. and H.H. conducted structure determination. J.T. and M.L. performed screening and enzyme assays with the help from Y.J.Z., B.H. and D.C. conducted cell-based assays as well as deubiquitylation and deISGylation experiments. Y.Y. and H.Z. assisted in X-ray data collection and data analysis. J.T. and Z.F. performed NMR experiments with the help from Z.L. and X.N., G.Z. and S.L. performed the antiviral and cytotoxicity assays. Y.J.Z. edited the manuscript. H.H. wrote the paper and everybody contributed to the writing.

\section{Competing interests}

A Chinese patent on the application of 6-TG and analogs to treating COVID-19 was filed on June 8, 2020 by Hao Huang, Guoliang Zhang, Jinle Tang, Shuyan Liu, Ziyang Fu and Ming Liu. The other authors declare no competing interests.

\section{Additional information}

Supplementary information is available for this paper at https://doi.org/10.1038/s41467020-20718-8.

Correspondence and requests for materials should be addressed to G.Z. or H.H.

Peer review information Nature Communications thanks the anonymous reviewer(s) for their contribution to the peer review of this work.

Reprints and permission information is available at http://www.nature.com/reprints

Publisher's note Springer Nature remains neutral with regard to jurisdictional claims in published maps and institutional affiliations.

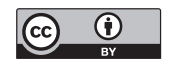

Open Access This article is licensed under a Creative Commons Attribution 4.0 International License, which permits use, sharing, adaptation, distribution and reproduction in any medium or format, as long as you give appropriate credit to the original author(s) and the source, provide a link to the Creative Commons license, and indicate if changes were made. The images or other third party material in this article are included in the article's Creative Commons license, unless indicated otherwise in a credit line to the material. If material is not included in the article's Creative Commons license and your intended use is not permitted by statutory regulation or exceeds the permitted use, you will need to obtain permission directly from the copyright holder. To view a copy of this license, visit http://creativecommons.org/ licenses/by/4.0/.

(C) The Author(s) 2021 